\title{
Bridging Methods for Atomistic-to-Continuum Coupling and Their Implementation
}

4 Pablo Seleson and Max Gunzburger*

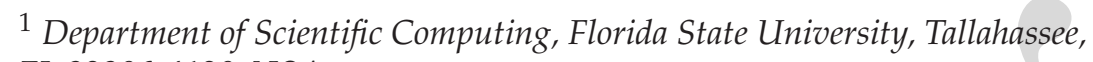

\begin{abstract}
Several issues connected with bridging methods for atomistic-to-continuum (AtC) coupling are examined. Different coupling approaches using various energy blending models are studied as well as the influence that model parameters, blending functions, and grids have on simulation results. We use the Lagrange multiplier method for enforcing constraints on the atomistic and continuum displacements in the bridge region. We also show that continuum models are not appropriate for dealing with problems with singular loads, whereas AtC bridging methods yield correct results, thus justifying the need for a multiscale method. We investigate models that involve multiple-neighbor interactions in the atomistic region, particularly focusing on a comparison of several approaches for dealing with Dirichlet boundary conditions.
\end{abstract}


same physical description, i.e., the same type of equations, on the whole domain; this is the case of the quasi-continuum method $[10,13,14]$. Other methods implement domain decomposition using different models in different domains, applying some sort of coupling mechanism between them; some examples of this type of approach are found in, e.g., $[1-4,8,15-17]$. For a review of multiscale material methods, the reader is referred to [18-20].

In atomistic-to-continuum (AtC) coupling techniques, an atomistic model is used in regions where microscale resolution is necessary but elsewhere, a (discretized) continuum model is applied. Several methods were proposed in the manner; for a comparison of different multiscale methods for the coupling of atomistic and continuum models see [9]. The central question in AtC coupling methods is how to couple the models, taking into account their different natures. In [1,2,4], a force-based blending model is applied to couple atomistic and continuum models. Blending is effected in a bridge region (also called interface or blending or overlap region) over which the atomistic displacement is constrained by the interpolation of the continuum displacement. Seemingly, such an approach over-constrains the system and causes the computational solution to deviate from what is expected.

Instead, we follow a similar approach to that in [3] and use a Lagrange multiplier method to enforce constraints, thus reducing the number of constraints. The focus in [3] is on a comparison between overlapping and non-overlapping domain decomposition methods, whereas we examine several components of overlapping domain decomposition methods (also called "handshake" models [12]) featuring two different blending schemes; we also study issues related to the implementation of those methods. In contrast to [1], where coupling is implemented at the force level, we blend the models at the energy level and use the minimization of the blended potential energy to determine the equilibrium configuration of the system; an approach, called the Arlequin method, for which the energy of the system is assumed to be shared between co-existent models was studied in [5-7]. This paper focuses on implementation details and difficulties of AtC coupling methods. In particular, we study several issues related to the application of an augmented Lagrange multiplier method, including the effects of nonuniformity of the Lagrange multiplier grid and the value chosen for the penalty parameter.

Another issue of interest is the application of boundary conditions. In physical systems, long-range interactions are the general case; therefore, multiple-neighbor interactions have to be implemented. Thus, an appropriate treatment is needed to correctly describe system interactions near the boundary where only a few atoms are available for interaction. In this paper, several different approaches for the case of Dirichlet, i.e., displacement, boundary conditions, in the context of multiple-neighbor interactions, are discussed and compared in Section 7.

The outline of this paper is as follows. In Section 2, we present the general framework of the AtC coupling method, as well as its implementation in one dimension. We describe the different components of the model as well as a physical interpretation for the energy blending technique. In Section 3, we introduce the quantitative tools implemented in the 
model analysis, as well as the different choices for some of the model components whose effects we investigate; in addition, we present an alternative energy blending model. In Section 4, we present a comparison between the energy blending models, and explain the correct application of the models to avoid the lack of partial interactions around the bridge boundaries. In Section 5, we study the convergence behavior of AtC blended models with respect to the different choices of the model components, including the blending functions, Lagrange multiplier basis, Lagrange multiplier grid, finite element grid, and penalty parameter value. In Section 6, we present singular load simulations showing that the finite element method is not appropriate for the treatment of regions with singular phenomena, whereas the AtC blended model produces correct results. In Section 7, we provide means for deriving model parameters for atomistic and AtC blended models for which one has multiple-neighbor atomistic interactions, and also compare the effectiveness of different ways to impose Dirichlet boundary conditions in such settings. We also show that for problems with singular loads, some anomalies can arise in computational solutions. Finally, in Section 8, we summarize the main conclusions of this research.

\section{The model}

\subsection{The general framework}

The general approach to find the equilibrium configuration of a system in the presence of external and internal forces involves the minimization of the total potential energy. Because we describe different parts of our domain $\Omega$ using different models, we need to find a way to combine both the continuum and the atomistic descriptions into a single potential energy expression. We define (in the reference configuration) three subregions: ${ }^{\dagger}$

$\Omega_{0}^{C}$ : the continuum region,

$\Omega_{0}^{M}$ : the atomistic region, $\Omega_{0}^{\text {bri }}=\Omega_{0}^{M} \cap \Omega_{0}^{C}$ : the bridge region.

Using blending functions, we determine the contribution to the global potential energy of each representation (continuum and atomistic) in each subregion.

We can express the total potential energy of the system ${ }^{\ddagger}$ as

$$
W=W^{\text {int }}-W^{\text {ext }}
$$

where we have the internal potential energy of the system

$$
W^{\text {int }}=\int_{\Omega_{0}^{C}} \xi(\mathbf{X}) w_{c} d \Omega_{0}^{C}+\frac{1}{2} \sum_{\alpha \in \mathcal{M}_{0}} \sum_{\beta \in \mathcal{N}_{\alpha}} \theta_{\alpha, \beta} w_{\alpha, \beta},
$$

${ }^{\mp}$ The atomistic model is assumed to be valid on the whole domain $\Omega$, but to make simulations tractable, is only used in the atomistic region $\Omega_{0}^{M}$; the continuum model is assumed to be valid only on $\Omega_{0}^{C}$.

$\ddagger$ We assume there are no time-dependent effects and our system is at zero temperature. 
and the external potential energy of the system

$$
W^{\mathrm{ext}}=\int_{\Omega_{0}^{C}} \xi(\mathbf{X}) \mathbf{B} \cdot \mathbf{u} d \Omega_{0}^{C}+\int_{\Gamma_{0}^{C}} \xi(\mathbf{X}) \mathbf{T} \cdot \mathbf{u} d \Gamma_{0}^{C}+\sum_{\alpha \in \mathcal{M}_{0}} \theta\left(\mathbf{X}_{\alpha}\right) \mathbf{f}_{\alpha}^{\mathrm{ext}} \cdot \mathbf{d}_{\alpha} .
$$

$\mathcal{M}_{0}=\left\{\alpha \mid \mathbf{X}_{\alpha} \in \Omega_{0}^{M}\right\}$ is the set of indexes of the atom positions in the atomistic region, and, for some given $\ell$ (which represents the length of the interatomic interaction), $\mathcal{N}_{\alpha}=$ $\left\{\beta\left|\mathbf{X}_{\beta} \in \Omega:\right| \mathbf{X}_{\beta}-\mathbf{X}_{\alpha} \mid<\ell\right\}$ is the set of indexes of the position of the atoms interacting with atom $\alpha ; w_{\alpha, \beta}$ is the potential energy of the atomistic bond $\alpha-\beta ; w_{c}=w_{c}(\mathbf{F})$ is the potential energy per unit volume of the continuum (as a function of the deformation gradient $\mathbf{F}$ ); $\mathbf{d}_{\alpha}=\mathbf{x}_{\alpha}-\mathbf{X}_{\alpha}$ is the displacement of the particle $\alpha \in \Omega_{0}^{M}$, with $\mathbf{x}_{\alpha}$ its position in the current configuration, and $\mathbf{X}_{\alpha}$ its position in the reference configuration; $\mathbf{u} \equiv \mathbf{u}(\mathbf{X})$ the continuum displacement of a point at $\mathbf{X} \in \Omega_{0}^{C} ; \mathbf{f}_{\alpha}^{\text {ext }}$ is the external force applied on the particle $\alpha ; \Gamma_{0}^{C}$ the boundary of $\Omega_{0}^{C}$; $\mathbf{B}$ the external body force (force per unit volume); $\mathbf{T}$ the boundary traction (the dependency of $\mathbf{B}, \mathbf{T}$, and $\mathbf{u}$ on $\mathbf{X}$ is omitted for simplicity); $\theta_{\alpha, \beta}$ an interatomic interaction blending function depending on $\theta(\mathbf{X}), \mathbf{X}_{\alpha}$, and $\mathbf{X}_{\beta}$. The energy blending functions $\xi(\mathbf{X})$ and $\theta(\mathbf{X})$ have the form

$$
\xi(\mathbf{X})=\left\{\begin{array}{cl}
0 & \mathbf{X} \in \Omega_{0}^{M} \backslash \Omega_{0}^{\text {bri }} \\
\alpha(\mathbf{X}) & \mathbf{X} \in \Omega_{0}^{\text {bri }} \\
1 & \mathbf{X} \in \Omega_{0}^{C} \backslash \Omega_{0}^{\text {bri }}
\end{array} \quad \text { and } \quad \theta(\mathbf{X})=\left\{\begin{array}{cl}
1 & \mathbf{X} \in \Omega_{0}^{M} \backslash \Omega_{0}^{\text {bri }} \\
1-\alpha(\mathbf{X}) & \mathbf{X} \in \Omega_{0}^{\text {bri }} \\
0 & \mathbf{X} \in \Omega_{0}^{C} \backslash \Omega_{0}^{\text {bri }}
\end{array}\right.\right.
$$

so that $\xi(\mathbf{X})+\theta(\mathbf{X})=1$, with $\alpha(\mathbf{X})$ a chosen function. The energy blending functions are used to divide the energy in the bridge region $\Omega_{0}^{\text {bri }}$ between the co-existent models, so that each model contributes partially to the total energy in the bridge region.

To apply displacement constraints between the continuum and atomistic descriptions in the bridge region, we use the augmented Lagrangian method; in addition to Lagrange multipliers for constraint enforcement, this method adds a penalty term to the potential energy. The total potential energy is replaced by the expression

$$
W_{A L}=W^{\mathrm{int}}-W^{\mathrm{ext}}+\lambda^{T} \cdot \mathbf{g}+\frac{1}{2} p \mathbf{g}^{T} \cdot \mathbf{g}
$$

with $\lambda=\left\{\lambda_{\gamma}\right\}$ a vector of Lagrange multipliers for the constraints and $\mathbf{g}=\left\{g_{\gamma}\right\}$ the constraint equation vector. Note that the penalty term is a positive quadratic form; thus, minimization renders this term small; $p$ is referred to as the penalty parameter.

\subsection{A linear one-dimensional case}

To better focus on the effects produced by the new approaches, we consider a simple onedimensional model with linear constraints. The expressions for the internal and external 
energies, i.e., (2.2) and (2.3) respectively, reduce to ${ }^{\S}$

$$
\begin{aligned}
& W^{\text {int }}=\int_{X_{i}}^{X_{f}} \xi(X) w_{c} d X+\frac{1}{2} \sum_{\alpha=1}^{N_{d}} \sum_{\beta \in \mathcal{N}_{\alpha}} \theta_{\alpha, \beta} w_{\alpha, \beta}, \\
& W^{\text {ext }}=\int_{X_{i}}^{X_{f}} \xi(X) \mathrm{B} u d X+\left.\xi(X) \mathrm{T} u\right|_{X_{i}} ^{X_{f}}+\sum_{\alpha=1}^{N_{d}} \theta\left(X_{\alpha}\right) f_{\alpha}^{\text {ext }} d_{\alpha},
\end{aligned}
$$

where $N_{d}$ is the number of particles in $\Omega_{0}^{M} ; X_{i}, X_{f}$ are the boundaries of the domain. The linear constraints over the bridge region are given by

$$
g_{\gamma}=u\left(X_{\gamma}\right)-d_{\gamma}=0 \quad \forall \gamma \in \mathcal{F} \equiv\left\{\alpha \mid X_{\alpha} \in \Omega_{0}^{\text {bri }}\right\},
$$

i.e., the constraints are applied to atomistic particles in the bridge region. To obtain the discrete equations, we implement a finite element (FE) method in $\Omega_{0}^{C}$ and a molecular mechanics description in $\Omega_{0}^{M}$; thus, we can express the approximate displacement field $u^{h}(X)$ in $\Omega_{0}^{C}$ in terms of the FE basis functions $\left\{\omega_{i}^{h}(X)\right\}, i=1, \cdots, N_{u}$, as follows:

$$
u(X) \approx u^{h}(X)=\sum_{i=1}^{N_{u}} \omega_{i}^{h}(X) u_{i}^{h}
$$

where the $u_{i}^{h}$ s denote the FE displacements at the FE nodes, and $N_{u}$ is the number of FE nodes in $\Omega_{0}^{C}$. The Lagrange multiplier (LM) field is also expressed in term of basis functions $\left\{\Lambda_{K}(X)\right\}, K=1, \cdots, N_{\lambda}$, (ultimately resulting in a reduction in the number of constraint equations)

$$
\lambda(X)=\sum_{K=1}^{N_{\lambda}} \Lambda_{K}(X) \bar{\lambda}_{K}
$$

with $\bar{\lambda}_{K}$ the LM coefficients and $N_{\lambda}$ the number of LM grid nodes in $\Omega_{0}^{\text {bri }}$. A general picture of our domain (assuming $\Omega_{0}^{M}=\left[X_{i}, c\right], \Omega_{0}^{C}=\left[a, X_{f}\right]$, and $\Omega_{0}^{\text {bri }}=[a, c]$ ) is presented in Fig. 1, with the thin red vertical bars representing the FE nodes, the thick green vertical bars the LM grid nodes, and the blue circles the atomistic particles.

Stable configurations of the AtC blended system are found by minimizing the energy (2.1), subject to the constraint (2.5), i.e., by solving the following set of equations:

$$
\frac{\partial W_{A L}}{\partial u_{j}^{h}}=0 ; j=1, \cdots, N_{u}, \quad \frac{\partial W_{A L}}{\partial d_{\zeta}}=0 ; \zeta=1, \cdots, N_{d}, \quad \frac{\partial W_{A L}}{\partial \lambda_{L}}=0 ; L=1, \cdots, N_{\lambda} .
$$

§The integrals in the continuum region $\Omega_{0}^{C}$ are extended to the entire domain, i.e., $\Omega=\left[X_{i}, X_{f}\right]$, since the energy blending function $\xi(X)$ vanishes outside $\Omega_{0}^{C}$. 


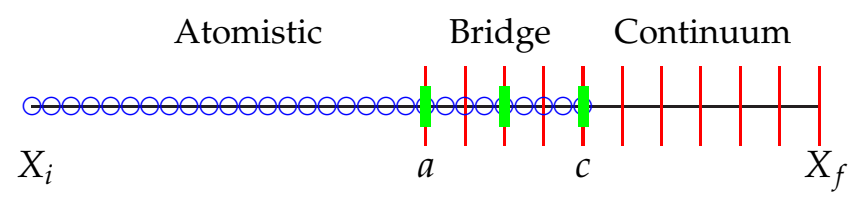

Figure 1: Atomistic-to-continuum coupling multiscale grid showing the atoms (blue circles), finite element nodes (thin red vertical bars) and Lagrange multiplier grid nodes (thick green vertical bars). Three regions are defined in the entire domain $\Omega=\left[X_{i}, X_{f}\right]$ : the atomistic region $\Omega_{0}^{M}=\left[X_{i}, c\right]$, the continuum region $\Omega_{0}^{C}=\left[a, X_{f}\right]$, and the bridge region $\Omega_{0}^{\mathrm{bri}}=[a, c]$ where both the atomistic and the continuum models co-exist.

The resulting system of equations is ${ }^{\mathbb{I}}$

$$
\begin{aligned}
& \int_{X_{i}}^{X_{f}} \xi(X) \frac{\partial w_{c}}{\partial u_{j}^{h}} d X+\sum_{\gamma \in \mathcal{F}}\left(\sum_{K=1}^{N_{\lambda}} \Lambda_{K}\left(X_{\gamma}\right) \bar{\lambda}_{K}\right) \omega_{j}^{h}\left(X_{\gamma}\right)+p \sum_{\gamma \in \mathcal{F}}\left(\sum_{i=1}^{N_{u}} \omega_{i}^{h}\left(X_{\gamma}\right) u_{i}^{h}-d_{\gamma}\right) \omega_{j}^{h}\left(X_{\gamma}\right) \\
& \quad=\int_{X_{i}}^{X_{f}} \xi(X) \mathrm{B}(X) \omega_{j}^{h}(X) d X+\left.\zeta(X) \mathrm{T}(X) \omega_{j}^{h}(X)\right|_{X_{i}} ^{X_{f}} \quad j=1, \ldots, N_{u} \\
& \frac{1}{2} \sum_{\alpha=1}^{N_{d}} \sum_{\beta \in \mathcal{N}_{\alpha}} \theta_{\alpha, \beta} \frac{\partial w_{\alpha, \beta}}{\partial d_{\zeta}}-\sum_{K=1}^{N_{\lambda}} \Lambda_{K}\left(X_{\zeta}\right) \bar{\lambda}_{K} \mathbf{I}_{\mathcal{F}}(\zeta)-p\left(\sum_{i=1}^{N_{u}} \omega_{i}^{h}\left(X_{\zeta}\right) u_{i}^{h}-d_{\zeta}\right) \mathbf{I}_{\mathcal{F}}(\zeta) \\
& =\theta\left(X_{\zeta}\right) f_{\zeta}^{\text {ext }}, \quad \zeta=1, \ldots, N_{d \prime} \\
& \sum_{\gamma \in \mathcal{F}} \Lambda_{L}\left(X_{\gamma}\right)\left(\sum_{i=1}^{N_{u}} \omega_{i}^{h}\left(X_{\gamma}\right) u_{i}^{h}-d_{\gamma}\right)=0, \quad L=1, \ldots, N_{\lambda}
\end{aligned}
$$

and $\mathcal{F}$ is defined in (2.5). Above and in the remainder of the paper we adopt the convention of using Greek subscripts, i.e., $\alpha, \beta, \gamma, \zeta$, to refer to atom numbers, Latin lowercase subscripts, i.e., $i, j$, to refer to FE node numbers, and Latin uppercase subscripts, i.e., $L, K$, to refer to LM node numbers.

There are several choices we have to make in order to implement our model; in the following, we present the main choices and relations for our model components.

where the indicator function

$$
\mathbf{I}_{\mathcal{F}}(\zeta)= \begin{cases}1 & \text { if } \zeta \in \mathcal{F}, \\ 0 & \text { if } \zeta \notin \mathcal{F},\end{cases}
$$

\footnotetext{
TIFor the unknowns corresponding to the displacements of the first atom and the last FE node, we replace
the equations by Dirichlet boundary conditions, i.e., $d_{1}=u\left(X_{1}\right)$ and $u_{N_{u}}^{h}=u\left(X_{N_{u}}^{h}\right)$ respectively, with $u(X)$ the
exact solution of our problem. In addition, in the case of nearest-neighbor interactions, the last atom of the
atomistic region of the AtC blended system, i.e., $\alpha=N_{d}$, is supposed to interact with a particle at the position
$X_{N_{d}+1} \equiv X_{N_{d}}+s$, where $s$ is the atomistic spacing; the position $X_{N_{d}+1}$ is in the continuum region beyond the
atomistic region of the AtC blended system, i.e., $\Omega_{0}^{C} \backslash \Omega_{0}^{\text {bri }}$, thus, an appropriate treatment in this case would
be to assume the displacement $d_{N_{d}+1}$ of a particle at $X_{N_{d}+1}$ is obtained by the interpolation of the continuum
approximation at that point (see Section 7.2.1). II For the unknowns corresponding to the displacements of the first atom and the last FE node, we replace
the equations by Dirichlet boundary conditions, i.e., $d_{1}=u\left(X_{1}\right)$ and $u_{N_{u}}^{h}=u\left(X_{N_{u}}^{h}\right)$ respectively, with $u(X)$ the
exact solution of our problem. In addition, in the case of nearest-neighbor interactions, the last atom of the
atomistic region of the AtC blended system, i.e., $\alpha=N_{d}$, is supposed to interact with a particle at the position
$X_{N_{d}+1} \equiv X_{N_{d}}+s$, where $s$ is the atomistic spacing; the position $X_{N_{d}+1}$ is in the continuum region beyond the
atomistic region of the AtC blended system, i.e., $\Omega_{0}^{C} \backslash \Omega_{0}^{\text {bri }}$, thus, an appropriate treatment in this case would
be to assume the displacement $d_{N_{d}+1}$ of a particle at $X_{N_{d}+1}$ is obtained by the interpolation of the continuum
approximation at that point (see Section 7.2.1).

TIFor the unknowns corresponding to the displacements of the first atom and the last FE node, we replace
the equations by Dirichlet boundary conditions, i.e., $d_{1}=u\left(X_{1}\right)$ and $u_{N_{u}}^{h}=u\left(X_{N_{u}}^{h}\right)$ respectively, with $u(X)$ the
exact solution of our problem. In addition, in the case of nearest-neighbor interactions, the last atom of the
atomistic region of the AtC blended system, i.e., $\alpha=N_{d}$, is supposed to interact with a particle at the position
$X_{N_{d}+1} \equiv X_{N_{d}}+s$, where $s$ is the atomistic spacing; the position $X_{N_{d}+1}$ is in the continuum region beyond the
atomistic region of the AtC blended system, i.e., $\Omega_{0}^{C} \backslash \Omega_{0}^{\text {bri }}$, thus, an appropriate treatment in this case would
be to assume the displacement $d_{N_{d}+1}$ of a particle at $X_{N_{d}+1}$ is obtained by the interpolation of the continuum
approximation at that point (see Section 7.2.1).

II For the unknowns corresponding to the displacements of the first atom and the last FE node, we replace
the equations by Dirichlet boundary conditions, i.e., $d_{1}=u\left(X_{1}\right)$ and $u_{N_{u}}^{h}=u\left(X_{N_{u}}^{h}\right)$ respectively, with $u(X)$ the
exact solution of our problem. In addition, in the case of nearest-neighbor interactions, the last atom of the
atomistic region of the AtC blended system, i.e., $\alpha=N_{d}$, is supposed to interact with a particle at the position
$X_{N_{d}+1} \equiv X_{N_{d}}+s$, where $s$ is the atomistic spacing; the position $X_{N_{d}+1}$ is in the continuum region beyond the
atomistic region of the AtC blended system, i.e., $\Omega_{0}^{C} \backslash \Omega_{0}^{\text {bri }}$, thus, an appropriate treatment in this case would
be to assume the displacement $d_{N_{d}+1}$ of a particle at $X_{N_{d}+1}$ is obtained by the interpolation of the continuum
approximation at that point (see Section 7.2.1).

TIFor the unknowns corresponding to the displacements of the first atom and the last FE node, we replace
the equations by Dirichlet boundary conditions, i.e., $d_{1}=u\left(X_{1}\right)$ and $u_{N_{u}}^{h}=u\left(X_{N_{u}}^{h}\right)$ respectively, with $u(X)$ the
exact solution of our problem. In addition, in the case of nearest-neighbor interactions, the last atom of the
atomistic region of the AtC blended system, i.e., $\alpha=N_{d}$, is supposed to interact with a particle at the position
$X_{N_{d}+1} \equiv X_{N_{d}}+s$, where $s$ is the atomistic spacing; the position $X_{N_{d}+1}$ is in the continuum region beyond the
atomistic region of the AtC blended system, i.e., $\Omega_{0}^{C} \backslash \Omega_{0}^{\text {bri }}$, thus, an appropriate treatment in this case would
be to assume the displacement $d_{N_{d}+1}$ of a particle at $X_{N_{d}+1}$ is obtained by the interpolation of the continuum
approximation at that point (see Section 7.2.1).

TIFor the unknowns corresponding to the displacements of the first atom and the last FE node, we replace
the equations by Dirichlet boundary conditions, i.e., $d_{1}=u\left(X_{1}\right)$ and $u_{N_{u}}^{h}=u\left(X_{N_{u}}^{h}\right)$ respectively, with $u(X)$ the
exact solution of our problem. In addition, in the case of nearest-neighbor interactions, the last atom of the
atomistic region of the AtC blended system, i.e., $\alpha=N_{d}$, is supposed to interact with a particle at the position
$X_{N_{d}+1} \equiv X_{N_{d}}+s$, where $s$ is the atomistic spacing; the position $X_{N_{d}+1}$ is in the continuum region beyond the
atomistic region of the AtC blended system, i.e., $\Omega_{0}^{C} \backslash \Omega_{0}^{\text {bri }}$, thus, an appropriate treatment in this case would
be to assume the displacement $d_{N_{d}+1}$ of a particle at $X_{N_{d}+1}$ is obtained by the interpolation of the continuum
approximation at that point (see Section 7.2.1).

TIFor the unknowns corresponding to the displacements of the first atom and the last FE node, we replace
the equations by Dirichlet boundary conditions, i.e., $d_{1}=u\left(X_{1}\right)$ and $u_{N_{u}}^{h}=u\left(X_{N_{u}}^{h}\right)$ respectively, with $u(X)$ the
exact solution of our problem. In addition, in the case of nearest-neighbor interactions, the last atom of the
atomistic region of the AtC blended system, i.e., $\alpha=N_{d}$, is supposed to interact with a particle at the position
$X_{N_{d}+1} \equiv X_{N_{d}}+s$, where $s$ is the atomistic spacing; the position $X_{N_{d}+1}$ is in the continuum region beyond the
atomistic region of the AtC blended system, i.e., $\Omega_{0}^{C} \backslash \Omega_{0}^{\text {bri }}$, thus, an appropriate treatment in this case would
be to assume the displacement $d_{N_{d}+1}$ of a particle at $X_{N_{d}+1}$ is obtained by the interpolation of the continuum
approximation at that point (see Section 7.2.1).

FE basis functions. We approximate the displacements of our system in the continuum

TIFor the unknowns corresponding to the displacements of the first atom and the last FE node, we replace
the equations by Dirichlet boundary conditions, i.e., $d_{1}=u\left(X_{1}\right)$ and $u_{N_{u}}^{h}=u\left(X_{N_{u}}^{h}\right)$ respectively, with $u(X)$ the
exact solution of our problem. In addition, in the case of nearest-neighbor interactions, the last atom of the
atomistic region of the AtC blended system, i.e., $\alpha=N_{d}$, is supposed to interact with a particle at the position
$X_{N_{d}+1} \equiv X_{N_{d}}+s$, where $s$ is the atomistic spacing; the position $X_{N_{d}+1}$ is in the continuum region beyond the
atomistic region of the AtC blended system, i.e., $\Omega_{0}^{C} \backslash \Omega_{0}^{\text {bri }}$, thus, an appropriate treatment in this case would
be to assume the displacement $d_{N_{d}+1}$ of a particle at $X_{N_{d}+1}$ is obtained by the interpolation of the continuum
approximation at that point (see Section 7.2.1).
} approximation at that point (see Section 7.21$)$. \begin{tabular}{l} 
\\
\\
\\
\\
\\
\\
\hline 139 \\
\hline 139 \\
\hline 139 \\
\hline 139 \\
\hline 139 \\
\hline
\end{tabular}

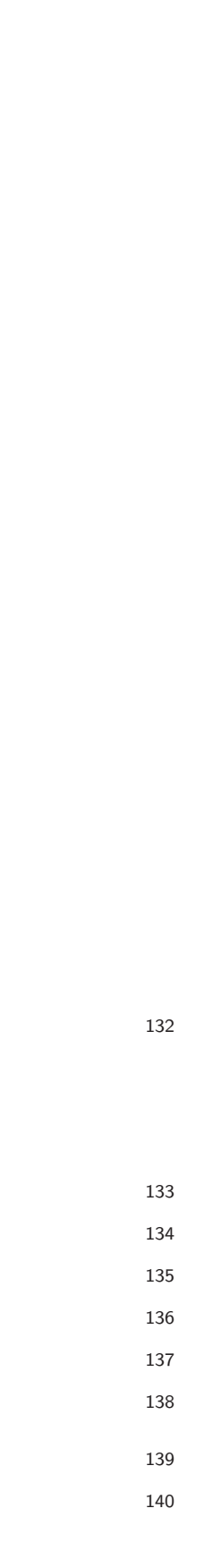


Discretized system. A more specific discretized system of equations is obtained by applying our continuum and atomistic interaction models (2.10) into the system of equations (2.8); then, we obtain, for the case of a nearest-neighbor interaction and piecewise linear FE basis functions, the discretized system of equations (2.11)-(2.13).

$$
\begin{aligned}
&\{\left.-K_{c}\left(\int_{X_{j-1}^{h}}^{X_{j}^{h}} \xi(X) d X\right) \frac{1}{\left(X_{j}^{h}-X_{j-1}^{h}\right)^{2}}+p \sum_{\gamma \in \mathcal{F}} \omega_{j-1}^{h}\left(X_{\gamma}\right) \omega_{j}^{h}\left(X_{\gamma}\right)\right\} u_{j-1}^{h} \\
&+\left\{K_{c}\left[\left(\int_{X_{j-1}^{h}}^{X_{j}^{h}} \xi(X) d X\right) \frac{1}{\left(X_{j}^{h}-X_{j-1}^{h}\right)^{2}}+\left(\int_{X_{j}^{h}}^{X_{j+1}^{h}} \xi(X) d X\right) \frac{1}{\left(X_{j+1}^{h}-X_{j}^{h}\right)^{2}}\right]\right. \\
&\left.+p \sum_{\gamma \in \mathcal{F}}\left(\omega_{j}^{h}\left(X_{\gamma}\right)\right)^{2}\right\} u_{j}^{h} \\
&+\left\{-K_{c}\left(\int_{X_{j}^{h}}^{X_{j+1}^{h}} \xi(X) d X\right) \frac{1}{\left(X_{j+1}^{h}-X_{j}^{h}\right)^{2}}+p \sum_{\gamma \in \mathcal{F}} \omega_{j+1}^{h}\left(X_{\gamma}\right) \omega_{j}^{h}\left(X_{\gamma}\right)\right\} u_{j+1}^{h} \\
&-p \sum_{\gamma \in \mathcal{F}} \omega_{j}^{h}\left(X_{\gamma}\right) d_{\gamma}+\sum_{K=1}^{N_{\lambda}}\left(\sum_{\gamma \in \mathcal{F}} \Lambda_{K}\left(X_{\gamma}\right) \omega_{j}^{h}\left(X_{\gamma}\right)\right) \bar{\lambda}_{K} \\
&=\int_{X_{i}}^{X_{f}} \xi(X) \mathrm{B}(X) \omega_{j}^{h}(X) d X+\left.\xi(X) \mathrm{T}(X) \omega_{j}^{h}(X)\right|_{X_{i}} ^{X_{f}} ; \quad j=1,2, \cdots, N_{u},
\end{aligned}
$$




$$
\begin{aligned}
& -\theta_{\alpha, \alpha-1}\left(\frac{K_{a}}{s}\right) d_{\alpha-1}+\left[\left(\theta_{\alpha, \alpha-1}+\theta_{\alpha, \alpha+1}\right)\left(\frac{K_{a}}{s}\right)+p \mathbf{I}_{\mathcal{F}}(\alpha)\right] d_{\alpha}-\theta_{\alpha, \alpha+1}\left(\frac{K_{a}}{s}\right) d_{\alpha+1} \\
& \quad-p \sum_{i=1}^{N_{u}} \omega_{i}^{h}\left(X_{\alpha}\right) u_{i}^{h} \mathbf{I}_{\mathcal{F}}(\alpha)-\sum_{K=1}^{N_{\lambda}} \Lambda_{K}\left(X_{\alpha}\right) \bar{\lambda}_{K} \mathbf{I}_{\mathcal{F}}(\alpha)=\theta_{\alpha} f_{\alpha}^{\mathrm{ext}} ; \quad \alpha=1,2, \cdots, N_{d}, \\
& \sum_{i=1}^{N_{u}}\left(\sum_{\gamma \in \mathcal{F}} \Lambda_{L}\left(X_{\gamma}\right) \omega_{i}^{h}\left(X_{\gamma}\right)\right) u_{i}^{h}-\sum_{\gamma \in \mathcal{F}} \Lambda_{L}\left(X_{\gamma}\right) d_{\gamma}=0 ; \quad L=1,2, \cdots, N_{\lambda},
\end{aligned}
$$

with $\theta_{\alpha} \equiv \theta\left(X_{\alpha}\right)$.

\subsection{A physical interpretation for energy blending}

So far we have presented a model based on energy blending. Once an energy blending form is imposed, the minimization of the potential energy provides the equilibrium configuration in the presence of external forces, given an internal potential energy function. In order to give a possible physical interpretation to our blending scheme, we start from a modified version of the continuum equilibrium equation, introducing the energy blending function $\xi(X)$ in a particular way as follows:

$$
\frac{d}{d X}(\xi(X) \mathrm{P}(X))+\xi(X) \mathrm{B}(X)=0 .
$$

In the case of hyperelastic materials, the Piola stress is given by $\mathbf{P}=\frac{\partial \Psi}{\partial \mathbf{F}}$, with $\Psi=\Psi(\mathbf{F})$ the strain-energy function and $\mathbf{F}=\mathbf{I}+\mathrm{Grad} \mathbf{u}$ the deformation gradient. In our onedimensional case,

$$
\Psi \equiv w_{c}=\frac{1}{2} K_{c}\left(\frac{d u}{d X}\right)^{2}=\frac{1}{2} K_{c}(F-1)^{2} ; \quad P=\frac{\partial \Psi}{\partial F}=K_{c}(F-1)=K_{c} \frac{d u}{d X} .
$$

Let us develop a numerical scheme using a FE method, starting from the equilibrium equation

$$
\frac{d}{d X}\left(\xi(X) K_{c} \frac{d u}{d X}\right)+\xi(X) \mathrm{B}(X)=0 .
$$

Multiplying by the test function $w_{j}^{h}(X)$, integrating and then using integration by parts, we obtain

$$
\int_{X_{i}}^{X_{f}} \xi(X) K_{c} \frac{d u}{d X}\left(w_{j}^{h}\right)^{\prime}(X) d X=\int_{X_{i}}^{X_{f}} \xi(X) \mathrm{B}(X) w_{j}^{h}(X) d X+\left.\xi(X) \mathrm{T}(X) w_{j}^{h}(X)\right|_{X_{i}{ }^{\prime}} ^{X_{f}}
$$

with $\mathrm{T}=K_{c} \frac{d u}{d X}$ the boundary traction (in the 1-D case, $\mathrm{T}=\mathrm{P}$ ). Assuming the displacement is approximated by a FE interpolation, i.e., $u(X) \approx u^{h}(X)$ as in (2.6), we can rewrite (2.16) as

$$
\int_{X_{i}}^{X_{f}} \xi(X) \frac{\partial w_{c}}{\partial u_{j}^{h}} d X=\int_{X_{i}}^{X_{f}} \xi(X) \mathrm{B}(X) w_{j}^{h}(X) d X+\left.\xi(X) \mathrm{T}(X) w_{j}^{h}(X)\right|_{X_{i}} ^{X_{f}} ;
$$


this is the contribution of the continuum model to the system (2.8). In (2.14), a blending was introduced in the physical quantities of the system; thus, it can be interpreted as a change in the elastic modulus, i.e., $K_{c} \rightarrow \xi(X) K_{c}$, and in the body force, i.e., $\mathrm{B}(X) \rightarrow$ $\xi(X) \mathrm{B}(X)$; therefore, those components change from a full contribution in the continuum region $\Omega_{0}^{C} \backslash \Omega_{0}^{\text {bri }}$ to a null contribution in the atomistic region $\Omega_{0}^{M} \backslash \Omega_{0}^{\text {bri }}$.

\section{Model analysis components}

Below, we analyze our model performance in terms of the errors of the AtC blended model when compared to the pure-atomistic one. We investigate the sensitivity of the results with respect to several components of our model, i.e.,

1. energy blending functions form in the bridge region: linear vs. cubic,

2. form of the interatomic interaction blending function,

3. Lagrange multiplier grid properties: uniformity and resolution,

4. Lagrange multiplier basis functions choice: piecewise linear vs. constant,

5. finite element grid resolution,

6. penalty parameter value.

For these purposes, we introduce, in Section 3.1, some quantitative tools. In Sections 3.23.5 , we provide different implementation choices for some of the model components, i.e., points 1-4 of the list above. Then, in Section 3.6, we discuss an alternative AtC blended model.

\subsection{Quantitative measurements}

Total error. We are interested in measuring the global error of our numerical simulations. Assuming the pure-atomistic model gives the correct solution for our system, we would like to calculate the error of the simulations produced by our AtC coupling methods in comparison to the pure-atomistic one. In the region $\left[X_{i}, c\right]$, we compare the displacements between the atoms in the pure-atomistic and the AtC blended models, whereas in $\left(c, X_{f}\right]$, the comparison is between the displacements of the atoms in the pure-atomistic model and the interpolation of the FE solution of the AtC blended models at the same positions. The calculation of the error $\epsilon$ is done using the $L_{2}$-like norm

$$
\epsilon=\left(\frac{1}{N} \sum_{\alpha=1}^{N_{d}}\left(d_{\alpha}-u_{\alpha}\right)^{2}+\frac{1}{N} \sum_{\alpha=N_{d}+1}^{N}\left(u^{h}\left(X_{\alpha}\right)-u_{\alpha}\right)^{2}\right)^{1 / 2},
$$

with $N$ the total number of atoms in $\left[X_{i}, X_{f}\right]$ in the pure-atomistic model, $N_{d}$ the number of atoms in $\left[X_{i}, c\right]$, i.e., the number of atoms in the atomistic region of the AtC blended model, $d_{\alpha}$ the displacement in the AtC blended model of the atom which was originally at the position $X_{\alpha}, u_{\alpha}$ the displacement in the pure-atomistic model of the atom originally at the position $X_{\alpha}$, and $u^{h}\left(X_{\alpha}\right)$ the FE approximation of the continuum displacement at the position $X_{\alpha}$. 
Cauchy strain. Deviations of the displacements for the case of smooth external forces can be relatively small in the sense that it is difficult for the eye to perceive them. An alternative way to visualize those deviations is through the slope of the displacements, i.e., the Cauchy strain. We measure the strain at $X_{i}$ as $e\left(X_{i}\right)=\left(u\left(X_{i+1}\right)-u\left(X_{i}\right)\right) /\left(X_{i+1}-\right.$ $\left.X_{i}\right)$. This is applied to the atomistic displacements of both the pure-atomistic and AtC blended models as well as to the FE approximation of the continuum displacement in the continuum domain of the AtC blended model.

\subsection{Energy blending functions}

One choice for the energy blending functions $\xi(X)$ and $\theta(X)$ is piecewise linear functions:

$$
\xi(X)=\left\{\begin{array}{cc}
0 & X<a \\
\frac{X-a}{c-a} & a \leq X \leq c \\
1 & X>c
\end{array}\right.
$$

with $\theta(X)=1-\xi(X)$. This choice is not $C^{1}$-continuous because the derivatives are discontinuous at the boundaries of the bridge region, i.e., at $X=a$ and $X=c$. To see if a smoother transition improves the accuracy of AtC simulations, we also use piecewise cubic functions with the requirement of $C^{1}$-continuity on $\left[X_{i}, X_{f}\right]$, i.e., $\xi(a)=0, \xi(c)=1, \xi^{\prime}(a)=0$, and $\xi^{\prime}(c)=0$. Using these conditions, we obtain the cubic energy blending function

$$
\xi(X)=\left\{\begin{array}{cc}
0 & X<a \\
\alpha x^{3}+\beta x^{2}+\gamma x+\delta & a \leq X \leq c \\
1 & X>c
\end{array}\right.
$$

with

$$
\alpha=-\frac{2}{(c-a)^{3}}, \quad \beta=-\frac{3}{2} \alpha(a+c), \quad \gamma=3 \alpha a c, \quad \delta=\frac{1}{2} \alpha a^{2}(a-3 c) .
$$

The reason we consider cubic as well as linear blending functions $\theta$ and $\xi$ is that the $C^{1}$ continuity of the cubic functions forces $\theta$ (respectively, $\xi$ ) to be small, relative to the linear case, in the bridge region near the continuum (respectively, atomistic) boundary $X=c$ (respectively, $X=a$ ). This results in a weakened effect of the atomistic (respectively, continuum) model in the bridge region near the continuum (respectively, atomistic) boundary which is perhaps desirable because presumably we want the continuum model to dominate in the bridge region near the continuum boundary and we certainly want the atomistic model to dominate in the bridge region near the atomistic boundary.

\subsection{Interatomic interaction blending function}

We use two different options for the interatomic interaction blending function $\theta_{\alpha, \beta}$ in the atomistic region; each one is consistent with a different integration rule for the integral, 
which contains the energy blending function $\xi(X)$, in the continuum portion of $W^{\text {int }}$. To show these relations, we start from the discretized continuum equation (2.11), set $p=$ 0 , and avoid the terms corresponding to the constraints, in order to focus only on the contributions from the internal and external energy expressions; the term containing the traction is neglected because we look at the internal nodes. The expression obtained is as follows:

$$
\begin{aligned}
& -K_{c}\left(\int_{X_{j-1}^{h}}^{X_{j}^{h}} \xi(X) d X\right) \frac{u_{j-1}^{h}-u_{j}^{h}}{\left(X_{j}^{h}-X_{j-1}^{h}\right)^{2}}-K_{c}\left(\int_{X_{j}^{h}}^{X_{j+1}^{h}} \xi(X) d X\right) \frac{u_{j+1}^{h}-u_{j}^{h}}{\left(X_{j+1}^{h}-X_{j}^{h}\right)^{2}} \\
= & \int_{X_{i}}^{X_{f}} \xi(X) \mathrm{B}(X) w_{j}^{h}(X) d X .
\end{aligned}
$$

Trapezoidal rule. Using the trapezoidal quadrature rule for the integrations, we get

$$
-K_{c}\left(\frac{\xi_{j-1}+\xi_{j}}{2}\right) \frac{u_{j-1}^{h}-u_{j}^{h}}{h}-K_{c}\left(\frac{\xi_{j}+\xi_{j+1}}{2}\right) \frac{u_{j+1}^{h}-u_{j}^{h}}{h}=\xi_{j} \mathrm{~B}\left(X_{j}^{h}\right) h,
$$

with $\xi_{j} \equiv \xi\left(X_{j}^{h}\right)$, where we have used a uniform FE grid with resolution $h$. Assuming $h=s$ and $j=\alpha$, we can write the equivalent expression for the atomistic interaction:

$$
-K_{a}\left(\frac{\theta_{\alpha-1}+\theta_{\alpha}}{2}\right)\left(\frac{d_{\alpha-1}-d_{\alpha}}{s}\right)-K_{a}\left(\frac{\theta_{\alpha}+\theta_{\alpha+1}}{2}\right)\left(\frac{d_{\alpha+1}-d_{\alpha}}{s}\right)=\theta_{\alpha} f_{\alpha}^{\text {ext }} .
$$

This gives some insight for the choice of $\theta_{\alpha, \beta}=\frac{1}{2}\left(\theta_{\alpha}+\theta_{\beta}\right)$; we refer to this interatomic interaction blending function approach as the "average" rule. Furthermore, we obtain the relations $K_{a}=K_{c}$ and $f_{\alpha}^{\text {ext }}=\mathrm{B}\left(X_{\alpha}\right) s$ that are implemented through our model.

Midpoint rule. We now consider (3.4) with a midpoint quadrature rule to approximate the integrals" to obtain

$$
-K_{c} \xi\left(\frac{X_{j-1}^{h}+X_{j}^{h}}{2}\right) \frac{u_{j-1}^{h}-u_{j}^{h}}{h}-K_{c} \xi\left(\frac{X_{j}^{h}+X_{j+1}^{h}}{2}\right) \frac{u_{j+1}^{h}-u_{j}^{h}}{h}=\xi\left(X_{j}\right) \mathrm{B}\left(X_{j}^{h}\right) h .
$$

Similarly to the "average" rule derivation, we obtain the "midpoint" rule for the interatomic interaction blending function, i.e., $\theta_{\alpha, \beta}=\theta\left(\frac{X_{\alpha}+X_{\beta}}{2}\right)$.

\subsection{Lagrange multiplier grid: uniformity and resolution}

We investigate the sensitivity of the AtC blended model on the number of LM grid nodes and the difference between the results obtained when using uniform and nonuniform

"We use the midpoint rule for the integrals on the left-hand side of (3.4), but still use the trapezoidal rule for the integral on the right-hand side. 


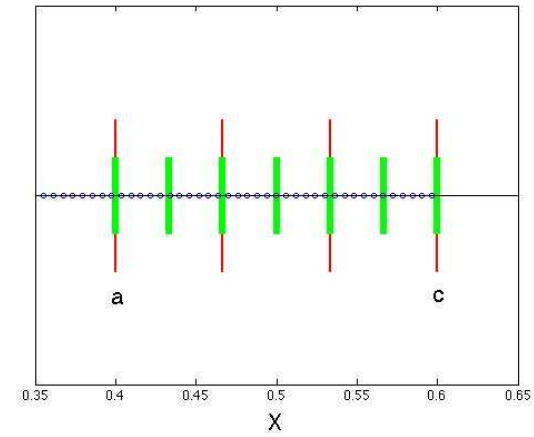

(a) Uniform LM grid

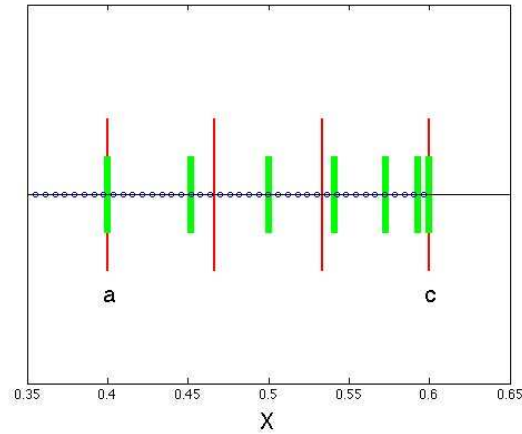

(b) Nonuniform LM grid

Figure 2: Comparison between a uniform (a) and a nonuniform (b) Lagrange multiplier (LM) grid. The LM grid nodes are represented by the thick green vertical bars; the thin red vertical bars correspond to finite element grid nodes, and the blue circles to atoms. The region $[a, c]$ represents the bridge $\Omega_{0}^{\text {bri }}$.

LM grids. The idea behind the implementation of a nonuniform LM grid is to strongly constrain the atomistic displacements by the continuum approximation near the continuum region $\Omega_{0}^{C} \backslash \Omega_{0}^{\text {bri }}$, whereas leaving the atoms less constrained close to the atomistic region $\Omega_{0}^{M} \backslash \Omega_{0}^{\text {bri }}$. To achieve that, we choose the number $N_{\lambda}$ of LM grid nodes; let $\Delta X=(c-a) /\left(N_{\lambda}-1\right)$, with $[a, c]$ the bridge region, and, for a uniform grid, simply choose the grid points

$$
X_{\lambda}(i)=a+\Delta X(i-1), \quad i=1, \cdots, N_{\lambda} .
$$

For the nonuniform grid, we apply a mapping to (3.5) so that

$$
\widetilde{X}_{\lambda}(i)=a+(c-a) \sin \left(\frac{\pi}{2} \frac{X_{\lambda}(i)-a}{c-a}\right), \quad i=1, \cdots, N_{\lambda}
$$

determines the LM grid points. In Fig. 2, we present, for illustration, a comparison between a uniform and a nonuniform LM grids, implemented on an AtC coupling multiscale grid with $N_{d}=100, N_{u}=10$, and $N_{\lambda}=7$; the bridge domain is $[a, c]=[0.4,0.6]$. In this example, equivalent pure-atomistic and pure-continuum FE grids would have 167 atoms and 16 nodes respectively, in the entire domain, i.e., $[0,1]$, resulting in 11 atoms per finite element.

\subsection{Lagrange multiplier basis functions choice: piecewise linear vs. constant}

We use two different approaches for the LM basis functions: piecewise constant and piecewise linear, and see how the results compare. Note that the number of LM basis functions is one less for the piecewise constant choice than for the piecewise linear choice, i.e., we have one less equation in our system. 


\subsection{Blending models}

Model I. The approach we started from is a blending of the energy in the form of (2.2)(2.3) with the total potential energy given by (2.1). We have shown, in the one-dimensional case, that, in the continuum, this is consistent with introducing the energy blending function $\xi(X)$ in the equilibrium equation as a change in the elastic modulus and in the body force as in (2.14). In [1], it was shown that a blending similar to the one presented in Model I leads to the satisfaction of Newton's third law.

To make the system of equations we are dealing with more clear, we write down the contributions of the internal and external potential energies to the discretized equations (2.11) and (2.12), i.e., we neglect the LM and penalty expressions. Using a trapezoidal rule for the integrals on the left-hand side (assuming a piecewise linear energy blending function $\xi(X)$ ) and correspondingly the "average" rule for the interatomic interaction blending function we obtain

$$
\begin{aligned}
& -\left(\frac{\xi_{j}+\xi_{j-1}}{2}\right) \frac{K_{c}}{\left(X_{j}^{h}-X_{j-1}^{h}\right)} u_{j-1}^{h}+\left[\left(\frac{\xi_{j}+\xi_{j-1}}{2}\right) \frac{K_{c}}{\left(X_{j}^{h}-X_{j-1}^{h}\right)}+\left(\frac{\xi_{j}+\xi_{j+1}}{2}\right) \frac{K_{c}}{\left(X_{j+1}^{h}-X_{j}^{h}\right)}\right] u_{j}^{h} \\
& -\left(\frac{\xi_{j}+\xi_{j+1}}{2}\right) \frac{K_{c}}{\left(X_{j+1}^{h}-X_{j}^{h}\right)} u_{j+1}^{h}=\int_{X_{i}}^{X_{f}} \xi(X) \mathrm{B}(X) \omega_{j}^{h}(X) d X+T\left(X_{f}\right) \omega_{j}^{h}\left(X_{f}\right) \\
& \quad-\left(\frac{\theta_{\alpha}+\theta_{\alpha-1}}{2}\right)\left(\frac{K_{a}}{s}\right) d_{\alpha-1}+\left[\left(\frac{\theta_{\alpha}+\theta_{\alpha-1}}{2}\right)+\left(\frac{\theta_{\alpha}+\theta_{\alpha+1}}{2}\right)\right]\left(\frac{K_{a}}{s}\right) d_{\alpha} \\
& -\left(\frac{\theta_{\alpha}+\theta_{\alpha+1}}{2}\right)\left(\frac{K_{a}}{s}\right) d_{\alpha+1}=\theta_{\alpha} f_{\alpha}^{\text {ext }} ; \quad \alpha=1,2, \cdots, N_{d} .
\end{aligned}
$$

In addition, the discretized continuum equation (3.6), in the limit of the atomistic resolution, assuming $K_{c}=K_{a}$ and $\mathrm{B}\left(X_{\alpha}\right) s=f_{\alpha}^{\text {ext }}$ (implementing the trapezoidal rule for integrating the right-hand side and neglecting the traction term), adds to the atomistic equation (3.7) to give the pure-atomistic force balance equation, i.e.,

$$
-\frac{K_{a}}{s} d_{\alpha-1}+2 \frac{K_{a}}{s} d_{\alpha}-\frac{K_{a}}{s} d_{\alpha+1}=f_{\alpha}^{\text {ext }} .
$$

Model II. A different approach for the blending of the continuum and atomistic models is obtained by letting the energy blending functions multiply the whole equilibrium equations. The continuum expression is

$$
\xi(X)\left\{\frac{d}{d X}(\mathrm{P}(X))+\mathrm{B}(X)=0\right\} .
$$

We multiply (3.9) by the test function $w_{j}^{h}(X)$ and integrate; using integration by parts and applying the explicit form of the nominal stress appearing in (2.15), i.e., $\mathrm{P}(X)=K_{c} \frac{d u}{d X}$, we 
get

$$
\begin{aligned}
& \int_{X_{i}}^{X_{f}} \xi(X) K_{c} \frac{d u}{d X}\left(w_{j}^{h}\right)^{\prime}(X) d X+\int_{X_{i}}^{X_{f}} K_{c} \frac{d u}{d X} \xi^{\prime}(X) w_{j}^{h}(X) d X \\
= & \int_{X_{i}}^{X_{f}} \xi(X) \mathrm{B}(X) w_{j}^{h}(X) d X+\left.\xi(X) \mathrm{T}(X) w_{j}^{h}(X)\right|_{X_{i}{ }^{\prime}} ^{X_{f}}
\end{aligned}
$$

that is similar to (2.16) but with an extra term, i.e., the second term on the left-hand side of (3.10). A discretization of (3.10) is

$$
\begin{aligned}
& -\xi_{j} \frac{K_{c}}{\left(X_{j}^{h}-X_{j-1}^{h}\right)} u_{j-1}^{h}+\xi_{j}\left[\frac{K_{c}}{\left(X_{j}^{h}-X_{j-1}^{h}\right)}+\frac{K_{c}}{\left(X_{j+1}^{h}-X_{j}^{h}\right)}\right] u_{j}^{h}-\xi_{j} \frac{K_{c}}{\left(X_{j+1}^{h}-X_{j}^{h}\right)} u_{j+1}^{h} \\
= & \int_{X_{i}}^{X_{f}} \xi(X) \mathrm{B}(X) \omega_{j}^{h}(X) d X+T\left(X_{f}\right) \omega_{j}^{h}\left(X_{f}\right) ; \quad j=1,2, \cdots, N_{u},
\end{aligned}
$$

assuming a piecewise linear energy blending function $\xi(X)$, and taking its derivative as $\xi^{\prime}(X)=\left(\xi_{j}-\xi_{j-1}\right) /\left(X_{j}^{h}-X_{j-1}^{h}\right)$ for $X \in\left(X_{j}^{h}, X_{j-1}^{h}\right)$. In the atomistic region, the corresponding expression is obtained by multiplying the pure-atomistic force balance equation (3.8) by $\theta_{\alpha}$. The resulting equation is

$$
-\theta_{\alpha} \frac{K_{a}}{S} d_{\alpha-1}+2 \theta_{\alpha} \frac{K_{a}}{s} d_{\alpha}-\theta_{\alpha} \frac{K_{a}}{s} d_{\alpha+1}=\theta_{\alpha} f_{\alpha}^{\text {ext }} ; \quad \alpha=1,2, \cdots, N_{d} .
$$

Notice that we have implemented the continuum and atomistic interaction models appearing in (2.10). In the case of a nearest-neighbor interaction, and assuming a FE grid resolution identical to the atomistic one, it is possible to show that Newton's third law is satisfied when taking into account both the atomistic and continuum force contributions to each atom/node. In particular, under those assumptions, adding the atomistic and discretized continuum expressions, i.e., (3.11) and (3.12), results in the pure-atomistic force balance equation (3.8).

The atomistic and continuum contributions to the equilibrium/force balance equations, both in Model I and Model II, add, under appropriate assumptions, to the pureatomistic force balance equation (3.8). This motivates the following statement. The AtC blended model can be reduced to a pure-continuum FE approximation with a nonuniform mesh. This can be obtained if we choose, in our AtC blended model, the same FE grid resolution in the bridge region, as in the atomistic model, i.e., $X_{j}^{h}-X_{j-1}^{h}=s$ for $X_{j}^{h}, X_{j-1}^{h} \in \Omega_{0}^{\text {bri }}$, and a particular quadrature rule for the right-hand side, so that the external forces in the continuum and atomistic models are the same (see, e.g., the trapezoidal rule in Section 6.2); in addition, we choose $K a=K_{c}$. In this case, the resulting model can also be obtained by using a FE method with a non-uniform mesh, with a resolution identical to the atomistic one in the atomistic region, i.e., in $\Omega_{0}^{M}$, while a different resolution is used in $\Omega_{0}^{C} \backslash \Omega_{0}^{\text {bri }}$ (equal to the one chosen for the FE approximation of the AtC blended model in that region). Whether a similar connection can be made in the case of a multiple-neighbor atomistic system and higher-order FE approximation, is an open question. 


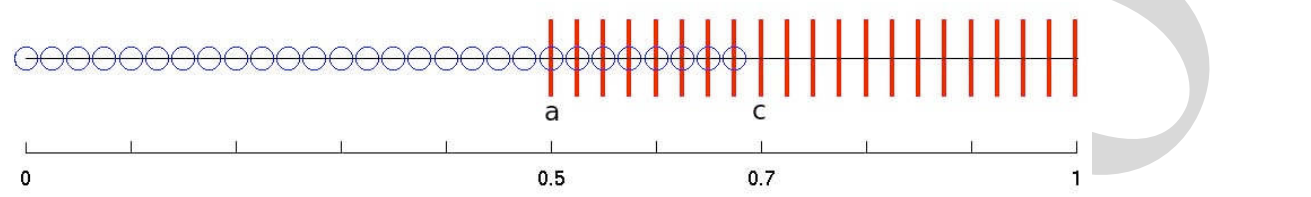

Figure 3: Multiscale grid composed of atoms (blue circles) and finite element (FE) nodes (red vertical bars). The FE grid resolution is identical to the atomistic one; there is no atom on the right boundary of the bridge region. The domain of the problem is $\Omega=[0,1]$, and the bridge region is chosen as $\Omega_{0}^{b r i}=[a, c]=[0.5,0.7]$.

\section{Comparing Model I and Model II in the bridge region}

In this section, we focus on the bridge boundaries, in order to point out some differences between Model I and Model II. For that purpose, we run a particular case for which the FE nodes overlap the atoms in the bridge region, but there is a missing atom at the last FE node of the bridge region, i.e., no atom is present on the right boundary of that region. The grid looks as in Fig. 3, with $21 \mathrm{FE}$ nodes in $\Omega_{0}^{C}=[0.5,1]$, and 28 atoms in $\Omega_{0}^{M}=[0,0.7]$. The atoms inside the bridge region correspond to atoms having the reference positions $\left\{X_{\alpha}\right\}, \alpha=21, \cdots, 28$, and the FE nodes that overlap them correspond to the FE nodes at $\left\{X_{j}^{h}\right\}, j=1, \cdots, 8$. We take a closer look at the assembled matrix for our discretized system of equations; in the assembled matrix $A$, the rows are ordered such that the first $N_{u}$ rows correspond to the FE equations, the following $N_{d}$ rows correspond to the atomistic equations, and the next $N_{\lambda}$ rows correspond to the constraints. We examine the resulting matrix after adding $A(42: 49,42: 49)+A(1: 8,1: 8)$, i.e., the sum of the contributions to the atoms/nodes in the bridge region; this contributions arise from the rows 1 to 8 , corresponding to the FE nodes in the bridge region (i.e., the first 8 FE nodes in $\Omega_{0}^{C}$ ) and from the rows 42 to 49 , corresponding to the atoms in the bridge region (i.e., the last 8 atoms in $\left.\Omega_{0}^{M}\right)$. In this numerical example, we used the following model choices: $N_{u}=21, N_{d}=28$, $N_{\lambda}=2, a=0.5, c=0.7, p=0$, piecewise linear $\xi(X)$, and piecewise linear $\Lambda_{L}(X)$. Models I and II both produce the matrix entries

$$
\left(\begin{array}{rrrrrrrr}
80 & -40 & 0 & 0 & 0 & 0 & 0 & 0 \\
-40 & 80 & -40 & 0 & 0 & 0 & 0 & 0 \\
0 & -40 & 80 & -40 & 0 & 0 & 0 & 0 \\
0 & 0 & -40 & 80 & -40 & 0 & 0 & 0 \\
0 & 0 & 0 & -40 & 80 & -40 & 0 & 0 \\
0 & 0 & 0 & 0 & -40 & 80 & -40 & 0 \\
0 & 0 & 0 & 0 & 0 & -40 & 80 & -40 \\
0 & 0 & 0 & 0 & 0 & 0 & -40 & 80
\end{array}\right) .
$$

In both models, the sum of the contributions of the continuum equilibrium equation and the atomistic force balance equation are consistent with the pure-atomistic interaction 
expression

$$
-\frac{K_{a}}{s} d_{\alpha-1}+2 \frac{K_{a}}{s} d_{\alpha}-\frac{K_{a}}{s} d_{\alpha+1}
$$

with $K_{a}=1.0$ and $s=1 / 40$; the total number of atoms in a pure-atomistic model having the same resolution is 41 .

Right boundary. We look at the assembled matrix $A$ of the system, but this time only at the continuum contributions. We focus on the elements corresponding to the first $10 \mathrm{FE}$ nodes of the continuum region, including eight overlapping FE nodes, a FE node at the right bridge boundary, and a FE node inside the continuum region outside the bridge region, i.e., $\Omega_{0}^{C} \backslash \Omega_{0}^{\text {bri }}$; this corresponds to the matrix block $A(1: 10,1: 10)$.

For Model I, we have the matrix block

$$
\left(\begin{array}{rrrrrrrrrr}
2.5 & -2.5 & 0 & 0 & 0 & 0 & 0 & 0 & 0 & 0 \\
-2.5 & 10 & -7.5 & 0 & 0 & 0 & 0 & 0 & 0 & 0 \\
0 & -7.5 & 20 & -12.5 & 0 & 0 & 0 & 0 & 0 & 0 \\
0 & 0 & -12.5 & 30 & -17.5 & 0 & 0 & 0 & 0 & 0 \\
0 & 0 & 0 & -17.5 & 40 & -22.5 & 0 & 0 & 0 & 0 \\
0 & 0 & 0 & 0 & -22.5 & 50 & -27.5 & 0 & 0 & 0 \\
0 & 0 & 0 & 0 & 0 & -27.5 & 60 & -32.5 & 0 & 0 \\
0 & 0 & 0 & 0 & 0 & 0 & -32.5 & 70 & -37.5 & 0 \\
0 & 0 & 0 & 0 & 0 & 0 & 0 & -37.5 & 77.5 & -40 \\
0 & 0 & 0 & 0 & 0 & 0 & 0 & 0 & -40 & 80
\end{array}\right) .
$$

The first 8 rows correspond to FE nodes that overlap atoms in the bridge region, thus we know that they have a complementary contribution from the atomistic expression (and we have verified that the continuum and atomistic contributions add together to the same pure-atomistic system interaction values). Row 9 is a FE node in the bridge domain (on the right boundary), but without an overlapping atom. Because of the energy blending function $\xi(X)$, the elements $A(9,8)=-37.5$ and $A(9,9)=77.5$ have values with smaller absolute value than those corresponding to the pure-atomistic model (which should be the same as the elements $A(10,9)=-40$ and $A(10,10)=80$, respectively), whereas there is no complimentary atomistic contribution.

For Model II, we have the matrix block

$$
\left(\begin{array}{rrrrrrrrrr}
0 & 0 & 0 & 0 & 0 & 0 & 0 & 0 & 0 & 0 \\
-5 & 10 & -5 & 0 & 0 & 0 & 0 & 0 & 0 & 0 \\
0 & -10 & 20 & -10 & 0 & 0 & 0 & 0 & 0 & 0 \\
0 & 0 & -15 & 30 & -15 & 0 & 0 & 0 & 0 & 0 \\
0 & 0 & 0 & -19 & 40 & -19 & 0 & 0 & 0 & 0 \\
0 & 0 & 0 & 0 & -25 & 50 & -25 & 0 & 0 & 0 \\
0 & 0 & 0 & 0 & 0 & -30 & 60 & -30 & 0 & 0 \\
0 & 0 & 0 & 0 & 0 & 0 & -35 & 70 & -35 & 0 \\
0 & 0 & 0 & 0 & 0 & 0 & 0 & -40 & 80 & -40 \\
0 & 0 & 0 & 0 & 0 & 0 & 0 & 0 & -40 & 80
\end{array}\right) .
$$


In this case, the FE node 9 has the correct interaction values.

In contrast to Model II, Model I suffers from an imbalance of the interaction values on the right boundary of the bridge region, because the blending is performed using an average of energy blending functions evaluated at different points; the lack of presence of an atom at the right boundary of the bridge region produces, as a result, only a partial contribution from the continuum model. Forcing the presence of an atom at the right boundary of the bridge region resolves this imbalance problem.

Left boundary. The problem appearing on the right boundary of the bridge region, corresponding to the system illustrated in Fig. 3, motivates a similar study in relation to the left boundary, but this time, for a general AtC configuration. An analogous problem to the one occurring at the right boundary of the bridge region, where the interaction arises only from a partial contribution of the continuum model, can occur on the left boundary of the bridge region; in particular, beyond it, but close to the bridge region, the atomistic contribution is only partial (because of the interatomic interaction blending function $\theta_{\alpha, \beta}$ ) and there is no additional contribution from the continuum model. Atoms inside the bridge region have partial contributions from each of the atomistic and continuum models; that is not the case for atoms outside the bridge region, which have only an atomistic contribution. In Model I, the interatomic interaction blending function $\theta_{\alpha, \beta}$ has an averaged form; thus, atoms outside the bridge region, but still interacting with atoms inside the bridge region, have an interaction weighted by $\theta_{\alpha, \beta}<1$, giving a smaller contribution in comparison to the pure-atomistic model.

In order to illustrate this, we examine the atomistic equation (3.7), for a given particle $\alpha$, where the "average" rule is used for $\theta_{\alpha, \beta}$. Assuming $X_{\alpha} \in\left[X_{i}, a\right]$ and $X_{\alpha+1} \in(a, c]$, then $\theta_{\alpha}=\theta_{\alpha-1}=1$, whereas $\theta_{\alpha+1}<1$. We then obtain

$$
-\left(\frac{K_{a}}{s}\right) d_{\alpha-1}+2\left(\frac{K_{a}}{s}\right) d_{\alpha}-\left(\frac{K_{a}}{s}\right) d_{\alpha+1}=f_{\alpha}^{\text {ext }}-\frac{1}{2}\left(1-\theta_{\alpha+1}\right)\left(\frac{K_{a}}{s}\right)\left(d_{\alpha+1}-d_{\alpha}\right) ;
$$

thus, an extra "artificial force" term appears on the right-hand side. A way to avoid this problem is to set the interatomic interaction blending function $\theta_{\alpha, \beta} \equiv 1$ in the atomistic force balance equation in (2.8) corresponding to atoms in the atomistic region outside the bridge region, i.e., atoms with an index $\zeta \notin \mathcal{F}$.

\section{Convergence studies}

In this section, we analyze, through computational experiments, the performance of models and their dependence on parameters, using two basic settings:
I. Zero-load case:
$\mathrm{B}(\mathrm{X})=0$;
$d_{1} \equiv u\left(X_{i}\right)=0$
$u_{N_{u}}^{h} \equiv u\left(X_{f}\right)=1$,
II. Constant-load case: $\mathrm{B}(X)=10 ; \quad d_{1} \equiv u\left(X_{i}\right)=0 ; \quad u_{N_{u}}^{h} \equiv u\left(X_{f}\right)=0$,

where $u(X)$ represents, in this case, the exact solution of our problem. In order to avoid boundary issues, we place an atom on the right boundary of the bridge region and use an interatomic interaction blending function $\theta_{\alpha, \beta} \equiv 1$ for atoms in the atomistic region outside 
the bridge region, i.e., $\Omega_{0}^{M} \backslash \Omega_{0}^{\text {bri }}$. The parameters used in common for the simulations in

Sections 5.1-5.3 are given in Table $1 .^{* *}$

Table 1: Model parameters for convergence studies.

\begin{tabular}{||c|c|c|c|c|c|c||}
\hline$N_{d}$ & $X_{i}$ & $a$ & $c$ & $X_{f}$ & $K_{c}$ & $K_{a}$ \\
\hline 129 & 0.0 & 0.4 & 0.64 & 1.0 & 1.0 & 1.0 \\
\hline
\end{tabular}

ॠ*The value of $c=0.64$ is chosen so that we obtain a FE node at the right boundary of the interface.

the AtC blended model (with an atomistic solution in $\left[X_{i}, C\right]$, and a continuum FE approximation in $\left.\left[a, X_{f}\right]\right)$, the pure-continuum FE model, and the exact solution of the PDE $-K_{c} \frac{d^{2} u}{d X^{2}}=\mathrm{B}(X)$ are presented in Fig. 4; a qualitative agreement is found between the different models. In the following sections, we investigate the error convergence as a function of different parameters, treating all possible combinations of the choices for the LM basis functions $\left(\Lambda_{L}(X)\right.$ piecewise linear or constant) and the energy blending function $(\xi(X)$ piecewise linear or cubic).

Figure 4: Comparison of the displacement profiles between the different models: atomistic-to-continuum (AtC) blended (with an atomistic solution in $\left[X_{i}, c\right]=[0,0.64]$, and a continuum finite element (FE) approximation in $\left.\left[a, X_{f}\right]=[0.4,1.0]\right)$, pure-atomistic, pure-continuum FE, and the PDE $-K_{c} \frac{d^{2} u}{d X^{2}}=B(X)$, for a zero-load case (a) and a constant-load case (b). The parameters used for the simulations are given in Table 1 along with $N_{\lambda}=7$, $N_{u}=21$, and $p=1$, using a uniform Lagrange multiplier (LM) grid for Model II; a piecewise cubic energy blending function $\xi(X)$ choice with the "average" rule (cf. Section 3.3) is implemented together with a piecewise linear LM basis functions $\Lambda_{L}(X)$. In addition to the displacement profiles, the multiscale grid is shown in the plots, with the blue circles representing atoms, the thin red vertical bars FE nodes, and the thick green vertical bars LM grid nodes. The plots show a qualitative agreement between the models. 


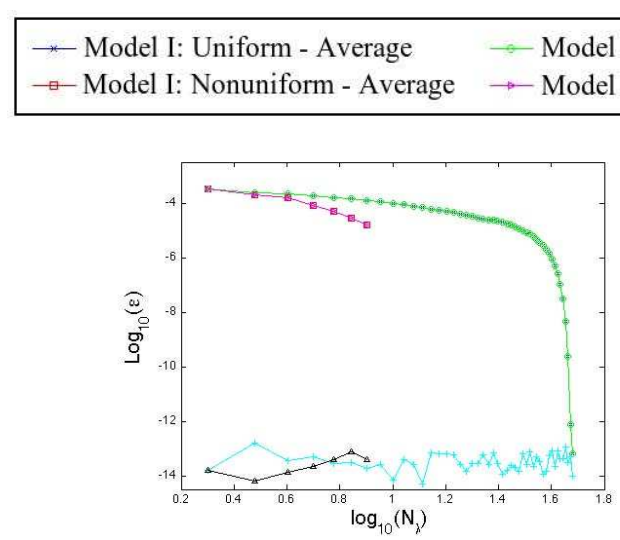

(a) $\Lambda_{L}(X)$ :p.linear - $\xi(X)$ : p.linear

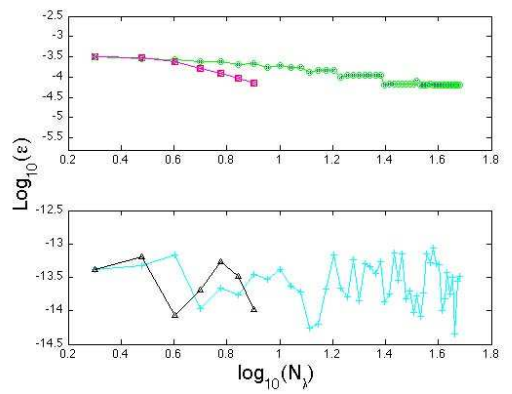

(c) $\Lambda_{L}(X)$ :p.const - $\xi(X)$ : p.linear

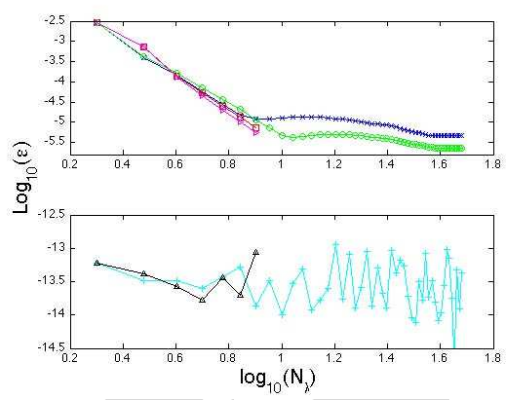

(b) $\Lambda_{L}(X):$ p.linear $-\xi(X)$ : p.cubic

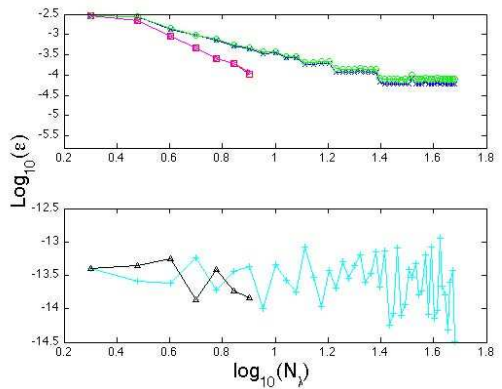

(d) $\Lambda_{L}(X)$ :p.const - $\xi(X)$ : p.cubic

Figure 5: Total error of the atomistic-to-continuum blended model as a function of the number of Lagrange multiplier (LM) grid nodes $N_{\lambda}$, for different cases, for the zero-load case. The plots (a, b, c, d) present different combinations for the choice of LM basis functions $\Lambda_{L}(X)$ : piecewise constant (p.const) or piecewise linear (p.linear), and the choice of the energy blending function $\xi(X)$ : piecewise linear (p.linear) or piecewise cubic (p.cubic). On each plot, the results of 6 simulations are shown, as it is described in the label of the figure; "uniform/nonuniform" refer to the LM grid (cf. Section 3.4), "midpoint/average" refer to the choice of the interatomic interaction blending function $\theta_{\alpha, \beta}$ (cf. Section 3.3), and "Model I/Model II" refer to the energy blending model ( $c f$. Section 3.6). Figures (b,c,d) use subplots because of the large differences in magnitude of the errors (y-axes) between the results of Model I and Model II (the results of Model I appear in the top plot of each subplot, whereas the results of Model II appear in the corresponding bottom plot). Notice that in $(a, c)$ there is an overlapping between the results of the "average" and "midpoint" rules, both in the case of a uniform and nonuniform LM grid, for Model I; as a consequence $(a, c)$ seem to present only 4 curves, instead of 6 . In (b,d) a similar behavior, though not identical, results from the "midpoint" and "average" rules in Model ।.

\subsection{Lagrange multiplier grid resolution}

We investigate the error behavior of the AtC blended model as a function of the number of LM grid nodes; we use in this case the values $N_{u}=21^{+\dagger}$ and $p=1$. For the zero-load case, the results are shown in Fig. 5; in Fig. 6, we present the results for the constant-load

${ }^{\text {F十 }}$ The values of $N_{d}=129$ (see Table 1 ) and $N_{u}=21$, correspond to resolutions equivalent to 201 atoms and 34 FE nodes in $[0,1]$; this choice gives a proportion of 6 atoms per finite element. 


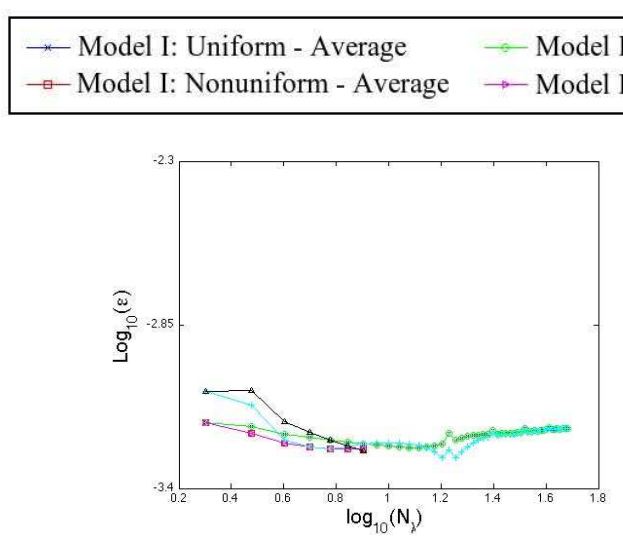

(a) $\Lambda_{L}(X)$ :p.linear - $\xi(X)$ : p.linear

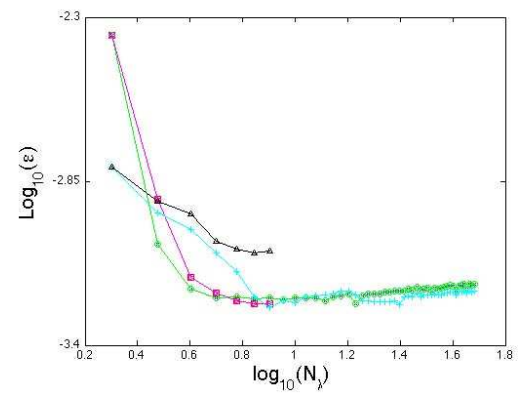

(c) $\Lambda_{L}(X)$ :p.const - $\xi(X)$ : p.linear

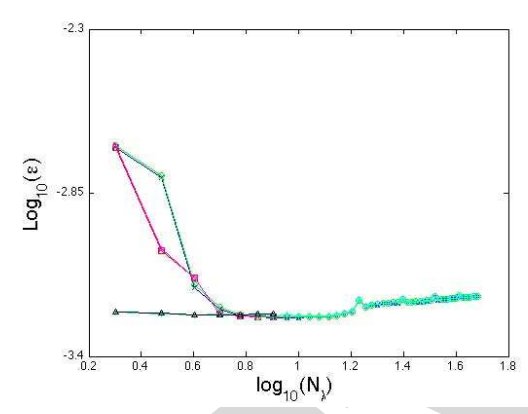

(b) $\Lambda_{L}(X)$ :p.linear $-\xi(X)$ : p.cubic

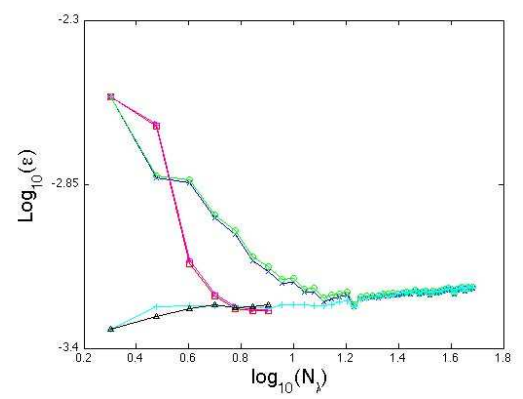

(d) $\Lambda_{L}(X):$ p.const - $\xi(X)$ : p.cubic

Figure 6: Total error of the atomistic-to-continuum blended model as a function of the number of Lagrange multiplier (LM) grid nodes $N_{\lambda}$, for different cases, for the constant-load case. The model choices and label interpretation are the same as in Fig. 5. As in the zero-load case (Fig. 5), in (a,c) there is an overlapping between the results of the "average" and "midpoint" rules, both in the case of a uniform and nonuniform LM grids, for Model I; in (b,d) a similar behavior, though not identical, results from the "midpoint" and "average" rules in Model I. Furthermore, in (b,d) similar results are obtained, for Model II, between a uniform and nonuniform LM grid choices; in particular, in (b) the curves overlap each other. As a consequence, the plots seem to present less than 6 curves.

case. The uniform and nonuniform LM grids follow the construction presented in Section 3.4; the maximum number of LM grid nodes taken in the simulations, both for the uniform $\left(N_{\lambda}=48\right)$ and nonuniform $\left(N_{\lambda}=8\right)$ LM grids, corresponds to the maximum number of nodes we can choose so that the LM grid resolution does not exceed the atomistic one.

The main conclusions are as follows.

- For the piecewise linear $\xi(X)$ choice (Figs. 5(a,c) and 6(a,c)), Model I produces the same results for the "average" and "midpoint" rules. The reason for that is that both rules are equivalent for linear functions.

- In the zero-load case, in contrast to Model I, (Fig. 5), Model II seems to produce the exact solution for all combinations (see the y-axes scales), similar to what happens in a pure-continuum FE case when the exact solution belongs to the FE space; however, in 
the constant-load case (Fig. 6), the errors for both models are of the same order. In order to understand the differences in behavior, we note that the displacement profile in the constant-load case has a quadratic form (see Fig. 4(b)), so that the choice of piecewiselinear interpolation for the FE method introduces an error of a similar magnitude in both models; in contrast, for the linear profile of the zero-load case, a piecewise-linear interpolation produces no errors.

- In the zero-load case (Fig. 5), for the piecewise linear $\xi(X)$ - piecewise linear $\Lambda_{L}(X)$ choice (a), Model I converges to the exact solution for a large number of LM grid nodes. - In the zero-load case (Fig. 5), for Model I, the nonuniform LM grid gives better convergence results $(a, c, d)$, though in the case of a piecewise cubic $\xi(X)$ - piecewise linear $\Lambda_{L}(X)$ choice (b), the difference between uniform and nonuniform LM grids is less noticeable. In the constant-load case (Fig. 6), for Model I, the use of a nonuniform LM grid does not improve the results in all the cases; on the other hand, in Model II, the uniform LM grid gives better results for the piecewise linear $\xi(X)$ choice $(a, c)$, whereas for the piecewise cubic $\xi(X)$ choice $(b, d)$, both LM grid choices produce similar results.

- In the zero-load case (Fig. 5), for the piecewise constant $\Lambda_{L}(X)$ choice (c,d), we see convergence, for the uniform LM grid case in Model I, including an approximate step-wise behavior of the solution.

- In the constant-load case (Fig. 6), in most of the cases, there seems to exist an optimal choice for $N_{\lambda}$ for which the error is minimized. That does not seem to be the case for Model II, for the piecewise cubic $\xi(X)$ choice, in particular, when using a piecewise constant LM basis functions $\Lambda_{L}(X)(\mathrm{d})$, where an approximate monotonic behavior for the error increase appears.

\subsection{Finite element grid resolution}

We next investigate the error behavior of the AtC blended model as a function of the number of FE nodes; for that purpose, we choose $N_{\lambda}=7$, i.e, $\log _{10}\left(N_{\lambda}\right) \approx 0.85$, which we observed in Section 5.1 gave a result close to optimal, in most of the cases, for the constant-load case (see Fig. 6), and $p=1$. The maximum number of FE nodes taken for the simulations is $N_{u}=121$ which gives a FE grid with the same resolution as the atomistic one. For the zero-load case, the results are shown in Fig. 7; in Fig. 8, we present the results for the constant-load case.

The main conclusions are as follows.

- For the piecewise linear $\xi(X)$ choice (Figs. $7(a, c)$ and $8(a, c))$, Model I produces the same results for the "average" and "midpoint" rules, as in Section 5.1.

- For the zero-load case (Fig. 7), Model II seems to produce the exact solution for all combinations (see the y-axes scales), in contrast to Model I, whereas in the constant-load case (Fig. 8), the errors for both models are of the same order, as in Section 5.1. On the contrary, in Fig. 7 (zero-load case) the errors in Model I seem to be independent of the FE grid resolution, and in Fig. 8 (constant-load case) the errors reach a plateau, whereas the errors in Model II continue decreasing when increasing the number of FE nodes. As in Sec- 


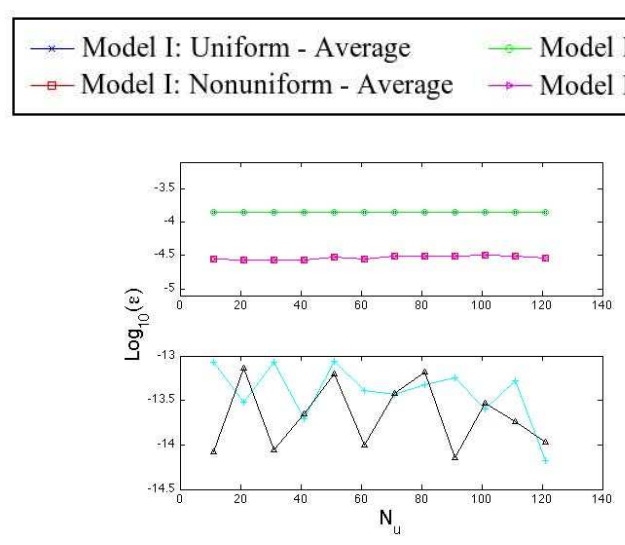

(a) $\Lambda_{L}(X)$ :p.linear $-\xi(X)$ : p.linear

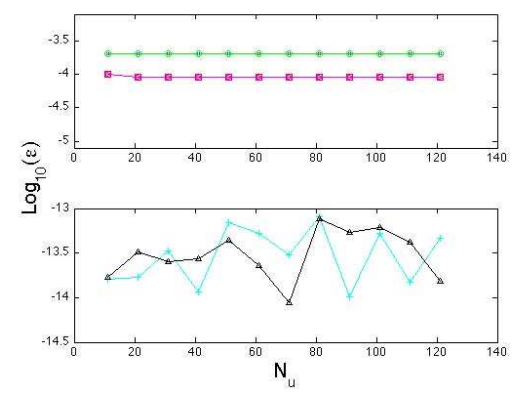

(c) $\Lambda_{L}(X)$ :p.const - $\xi(X)$ : p.linear

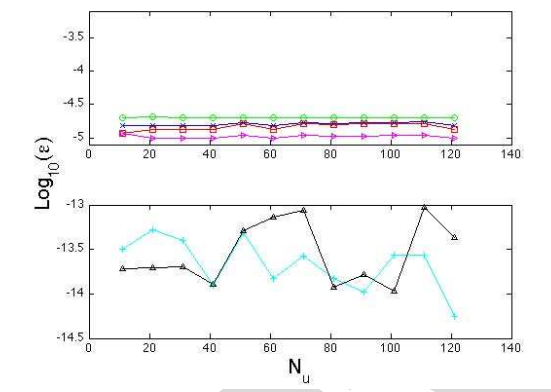

(b) $\Lambda_{L}(X):$ p.linear $-\xi(X)$ : p.cubic

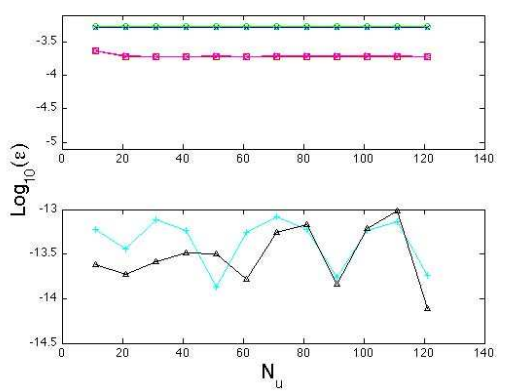

(d) $\Lambda_{L}(X)$ :p.const - $\xi(X)$ : p.cubic

Figure 7: Total error of the atomistic-to-continuum blended model as a function of the number of finite element grid nodes $N_{u}$, for different cases, for the zero-load case. The model choices and label interpretation are the same as in Fig. 5. Figures $(a, b, c, d)$ use subplots because of the large differences in magnitude of the errors ( $y$ axes) between the results of Model I and Model II (the results of Model I appear in the top plot of each subplot, whereas the results of Model II appear in the corresponding bottom plot). In (a,c), there is an overlapping between the results of the "average" and "midpoint" rules, both in the case of a uniform and nonuniform Lagrange multiplier grids, for Model I; as a consequence $(a, c)$ seem to present only 4 curves, instead of 6 . In $(b, d)$ a similar behavior, though not identical, results from the "midpoint" and "average" rules in Model I.

tion 5.1, we can argue that the piecewise linear FE approximation introduces additional errors in the constant-load case, so that both models have errors of the same magnitude; on the other hand, it is clear that an additional source of error is present in Model I and becomes dominant for a large number of FE nodes. A possible interpretation is that there is some error arising from noise in the bridge region that prevents convergence. To show the noise created in the bridge region, we present, in Fig. 9, a plot comparing the strain profiles for Model I (a) and Model II (b).

- In Model I, the nonuniform LM grid gives lower errors than the uniform one (see Figs. 7 and 8).

- In the constant-load case (Fig. 8), for piecewise cubic $\xi(X)$ choice (b,d), Model II produces smaller errors than Model I; that is not exactly the case for the piecewise linear $\xi(X)$ 


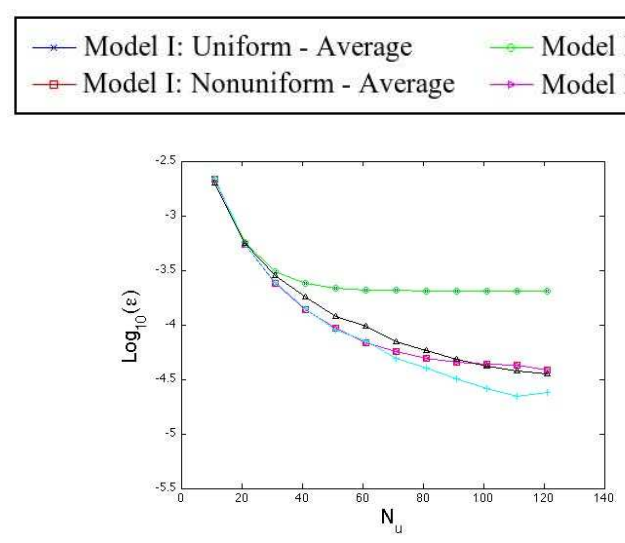

(a) $\Lambda_{L}(X)$ :p.linear - $\xi(X)$ : p.linear

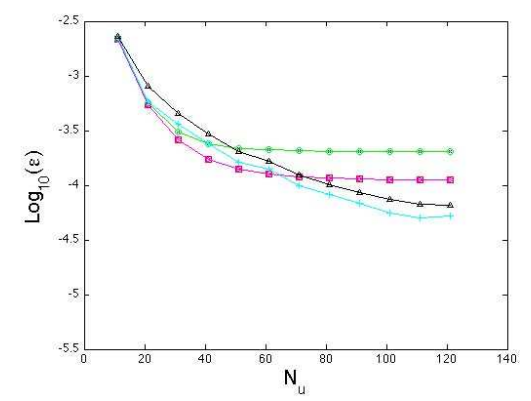

(c) $\Lambda_{L}(X)$ :p.const $-\xi(X)$ : p.linear

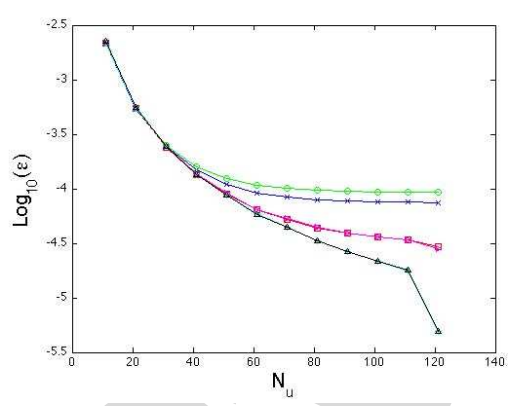

(b) $\Lambda_{L}(X)$ :p.linear - $\xi(X)$ : p.cubic

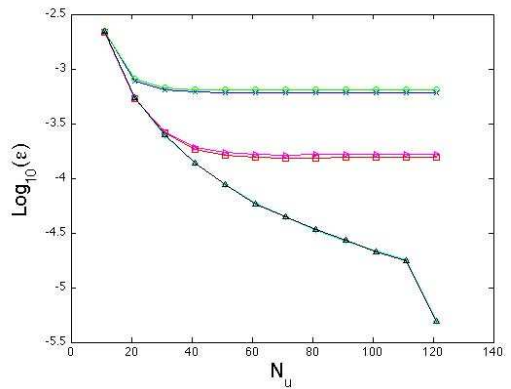

(d) $\Lambda_{L}(X)$ :p.const - $\xi(X)$ : p.cubic

Figure 8: Total error of the atomistic-to-continuum blended model as a function of the number of finite element grid nodes $N_{u}$, for different cases, for the constant-load case. The model choices and label interpretation are the same as in Fig. 5. As in the zero-load case (Fig. 7), there is an overlapping between some of the curves presented in the results; in $(a, c)$, there is an overlapping between the results of the "average" and "midpoint" rules, both in the case of a uniform and nonuniform Lagrange multiplier (LM) grids, for Model I; in (b,d) a similar behavior, though not identical, results from the "midpoint" and "average" rules in Model I. Furthermore, in (b,d), for Model II, there is an overlapping between the results of a uniform and nonuniform LM grid choices. As a consequence, the plots seem to present less than 6 curves.

choice $(a, c)$.

- In Model II, in the constant-load case (Fig. 8), we get the same results for a uniform and nonuniform LM grids in the case of a piecewise cubic $\xi(X)$ choice $(b, d)$, whereas a uniform LM grid performs better than the nonuniform one in the case of a piecewise linear $\xi(X)$ choice $(a, c)$.

\subsection{Penalty parameter}

We investigate the error, of the AtC blended model, as a function of the penalty parameter. We choose $N_{\lambda}=7$, as in Section 5.2, and $N_{u}=21$. For the zero-load case, results are shown in Fig. 10, whereas in Fig. 11 the results in a semi-log scale show the asymptotic 


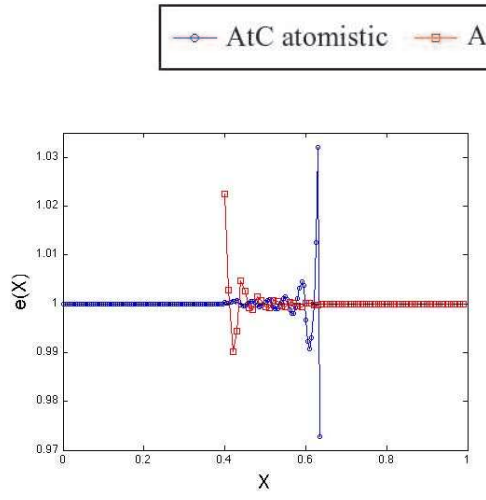

(a) Model I

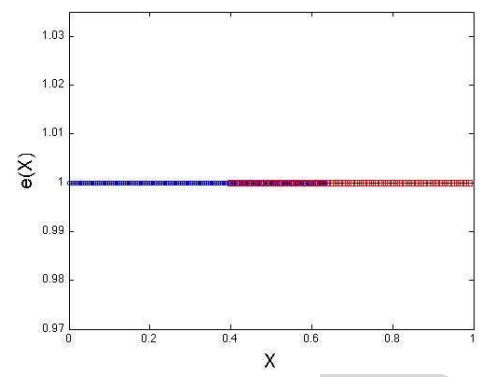

(b) Model II

Figure 9: Comparison of the strain profiles between the atomistic-to-continuum (AtC) blended model (with an atomistic solution in $[0,0.64]$, and a continuum finite element approximation in $[0.4,1.0]$ ), and the pure-atomistic model, for the zero-load case, in Model I (a) and Model II (b) (cf. Section 3.6). The simulation parameters are presented in Table $1 ; N_{\lambda}=7, N_{u}=61, p=1$; we use a piecewise cubic energy blending function $\xi(X)$ choice, the "average" rule (cf. Section 3.3) for Model I, a piecewise linear Lagrange multiplier (LM) basis functions $\Lambda_{L}(X)$ choice, and a uniform LM grid.

behavior for large values of $p$; in Fig. 12, we present the results for the constant-load case, whereas in Fig. 13 the results in a semi-log scale show the asymptotic behavior for large values of $p$.

The main conclusions are as follows.

- In the piecewise linear $\xi(X)$ choice (Figs. 10(a,c), 11(a,c), 12(a,c), and 13(a,c)), Model I produces the same results for the "average" and "midpoint" rules, as in Sections 5.1 and 5.2 .

- In the zero-load case (Figs. 10 and 11), Model II seems to produce the exact solution for all combinations (see the y-axes scales), in contrast to Model I, whereas in the constantload case (Figs. 12 and 13), the errors for both models are of the same order, as in Sections 5.1 and 5.2 .

- In the zero-load case (Figs. 10 and 11), for Model I, the error seems to decrease monotonically with increasing $p$; furthermore, the nonuniform LM grid, in most of the cases, results in a smaller error than the uniform one.

- In the constant-load case (Figs. 12 and 13), there exists an optimal value of $p \approx 40$ for the error convergence in the case of a piecewise linear $\xi(X)$ choice $(a, c)$ in Model II, whereas in the piecewise cubic $\xi(X)$ choice $(b, d)$ the error basically increases with increasing $p$; in Model I, the optimal value for $p$ in the piecewise linear $\xi(X)$ case is around 10.

- In the constant-load case (Figs. 12 and 13), for the piecewise cubic $\xi(X)$ choice (b,d), after some value of $p$, the behavior is similar for both models. On the contrary, for the piecewise linear $\xi(X)$ choice $(\mathrm{a}, \mathrm{c})$, after some value of $p$, Model II outperforms Model I. - In the constant-load case (Figs. 12 and 13), the minimum value for the error obtained in the piecewise linear $\xi(X)$ choice $(a, c)$ is smaller than that obtained in the piecewise cubic $\xi(X)$ choice $(b, d)$. 


\begin{tabular}{lll}
\hline$\rightarrow$ Model I: Uniform - Average & $\rightarrow$ Model I: Uniform - Midpoint & $\rightarrow$ Model II: Uniform \\
$\rightarrow-$ Model I: Nonuniform - Average & $\rightarrow$ Model I: Nonuniform - Midpoint $\rightarrow$ Model II: Nonuniform \\
\hline
\end{tabular}

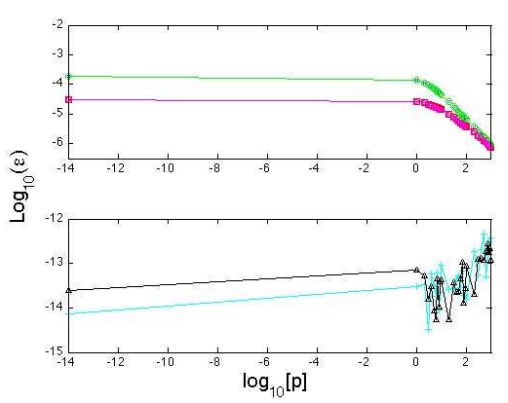

(a) $\Lambda_{L}(X)$ :p.linear - $\xi(X)$ : p.linear

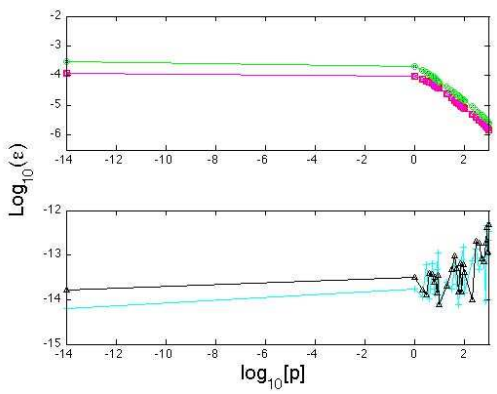

(c) $\Lambda_{L}(X)$ :p.const - $\xi(X)$ : p.linear

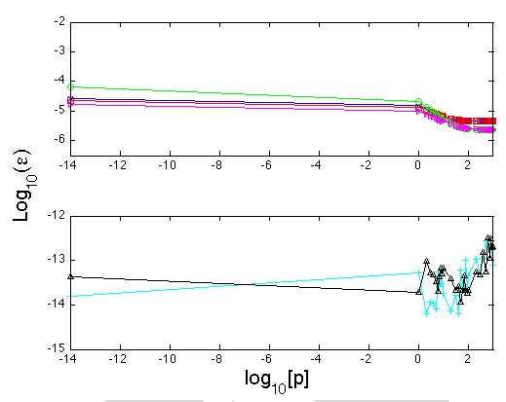

(b) $\Lambda_{L}(X):$ p.linear $-\xi(X)$ : p.cubic

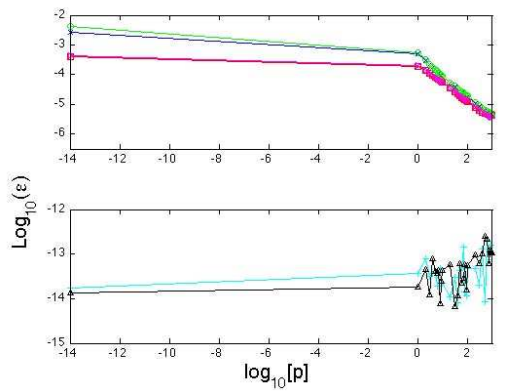

(d) $\Lambda_{L}(X)$ :p.const - $\xi(X)$ : p.cubic

Figure 10: Total error of the atomistic-to-continuum blended model as a function of the penalty parameter $p$, for different cases, for the zero-load case. The model choices and label interpretation are the same as in Fig. 5. Figures $(a, b, c, d)$ use subplots because of the large differences in magnitude of the errors ( $y$-axes) between the different cases. In $(a, c)$, there is an overlapping between the results of the "average" and "midpoint" rules, both in the case of a uniform and nonuniform Lagrange multiplier grids, for Model I; as a consequence $(a, c)$ seem to present only 4 curves, instead of 6 . In (b,d) a similar behavior, though not identical, results from the "midpoint" and "average" rules in Model I.

\subsection{Main conclusions regarding the error convergence}

Following the above results we can arrive at the following general conclusions.

- Model II outperforms Model I (or is at least as good) for most cases. In particular, for the zero-load case, Model II seems to reproduce the exact solution.

- Linear energy blending (piecewise linear $\xi(X)$ choice), applied to Model I, does not distinguish between the "average" and "midpoint" rules.

- For Model I, nonuniform LM grids result, in most of the cases, in smaller errors compared to uniform LM grids; on the contrary, for Model II, better results are obtained, in most of the cases, when applying a uniform LM grid.

- In several cases (in particular for the constant-load simulations), optimal values for $N_{\lambda}$ and $p$ are found. 


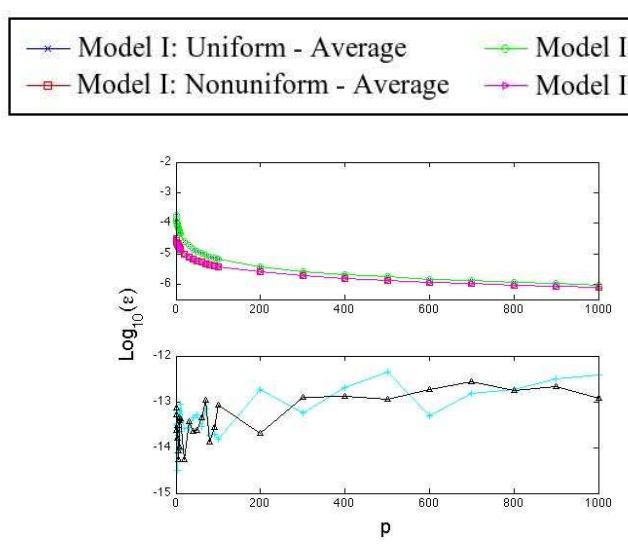

(a) $\Lambda_{L}(X)$ :p.linear $-\xi(X)$ : p.linear

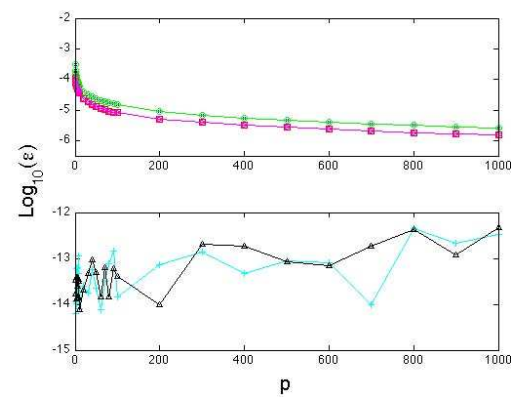

(c) $\Lambda_{L}(X)$ :p.const - $\xi(X)$ : p.linear

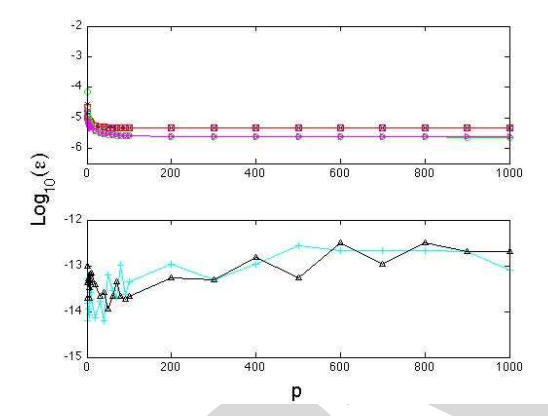

(b) $\Lambda_{L}(X):$ p.linear $-\xi(X)$ : p.cubic

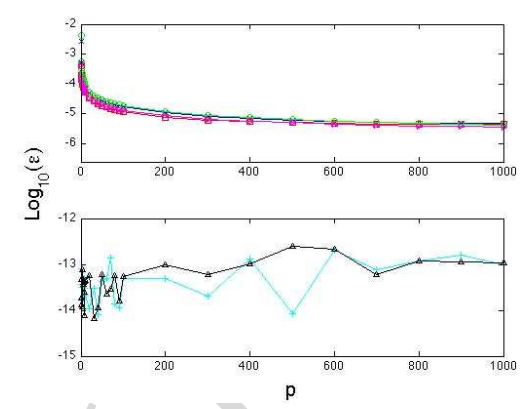

(d) $\Lambda_{L}(X)$ :p.const - $\xi(X)$ : p.cubic

Figure 11: Total error of the atomistic-to-continuum blended model as a function of the penalty parameter $p$, for different cases, for the zero-load case in a semi-log scale. The model choices and label interpretation are the same as in Fig. 5. As in Fig. 10, we use subplots, and, in addition, some curves overlap each other (see the caption of Fig. 10 for further details).

\section{Singular load}

So far we have shown that the AtC blended models reproduce pretty well results obtained using the pure-atomistic model; see, e.g., Fig. 4. The results obtained so far were for smooth exact solutions that can be solved much more cheaply using a pure-continuum FE model; in fact, in Fig. 4, we see that the pure-continuum FE model also yields good solutions. Recall that the motivation for developing the AtC coupling methods is to treat problems that cannot be accurately approximated with just a FE model. In this section, we apply the different models (AtC blended, pure-atomistic, pure-continuum FE, and the PDE $\left.-K_{c} \frac{d^{2} u}{d X^{2}}=B(X)\right)$ to a problem with a singular load, i.e., a force that is applied only on a few atoms, for which we expect that the FE method does not yield accurate results.

To compare the performance of the pure-continuum FE model with the AtC blended model, in the case of a singular load, we need to relate a discrete force, acting at the microscopic level, with a continuous body force, acting at the macroscopic level, in such 


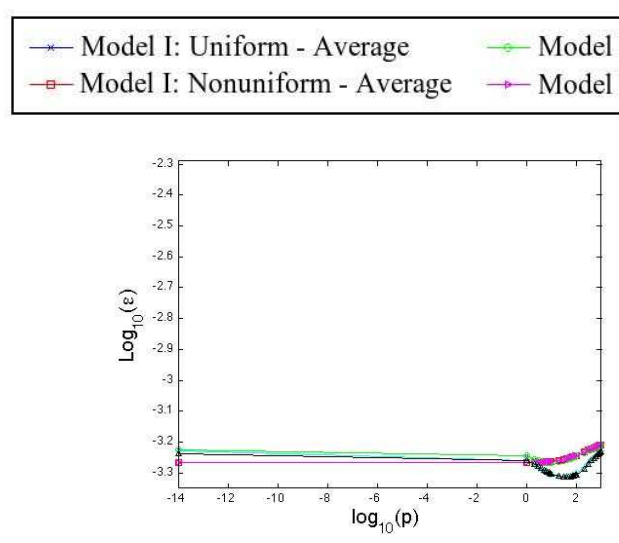

(a) $\Lambda_{L}(X)$ :p.linear - $\xi(X)$ : p.linear

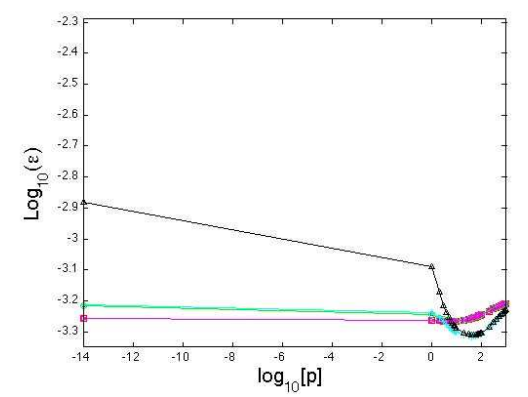

(c) $\Lambda_{L}(X)$ :p.const $-\xi(X)$ : p.linear

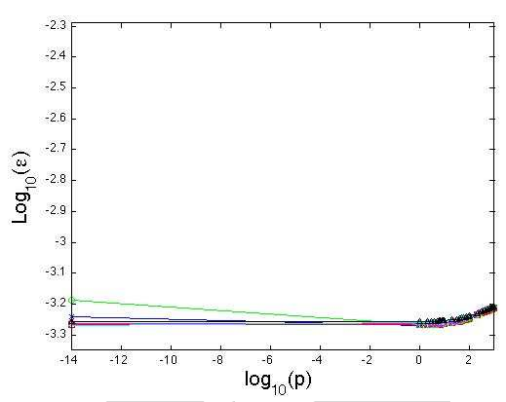

(b) $\Lambda_{L}(X)$ :p.linear - $\xi(X)$ : p.cubic

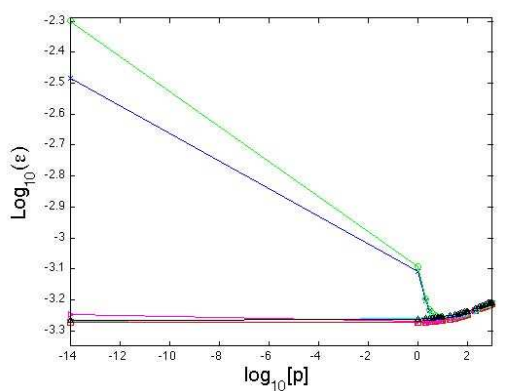

(d) $\Lambda_{L}(X)$ :p.const - $\xi(X)$ : p.cubic

Figure 12: Total error of the atomistic-to-continuum blended model as a function of the penalty parameter $p$, for different cases, for the constant-load case. The model choices and label interpretation are the same as in Fig. 5. As in the zero-load case (Figs. 10 and 11), some curves overlap each other; in $(a, c)$, there is an overlapping between the results of the "average" and "midpoint" rules, both in the case of a uniform and nonuniform Lagrange multiplier (LM) grids, for Model I; in (b,d) a similar behavior, though not identical, results from the "midpoint" and "average" rules in Model I. Furthermore, in (b,d) a similar behavior results for the uniform and nonuniform LM grid choices, in Model II. As a consequence, some plots seem to present less that 6 curves. Notice that the y-scale was stretched out in comparison to Figs. 10 and 11.

a way that a consistent application of the same force source is effected in both cases. Otherwise, we would be comparing systems with different external forces. The next two sections present a discussion about this issue.

\subsection{From a continuous to a discrete force}

Assume we are given a continuous body force function. We would like to zoom in to a particular region of our system, and ask what is the corresponding force acting on individual particles. In particular, when the force is very sharp, i.e., nonzero only on a few particles, we would like to determine the right contribution for each one. The situation is sketched in Fig. 14; on the left, the contribution of the continuous force profile 


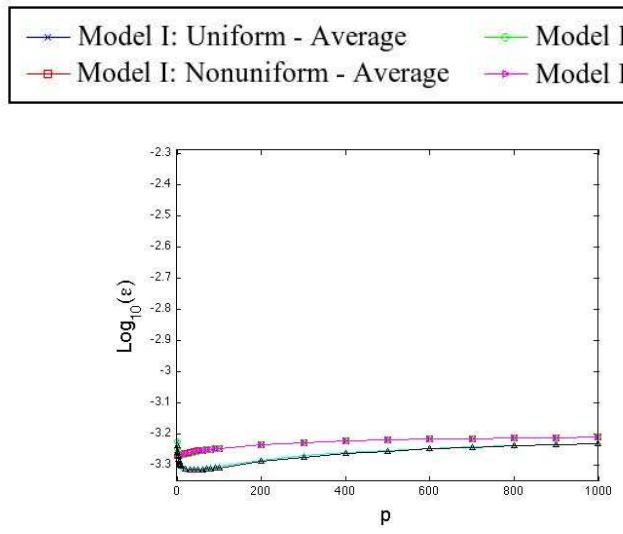

(a) $\Lambda_{L}(X)$ :p.linear - $\xi(X)$ : p.linear

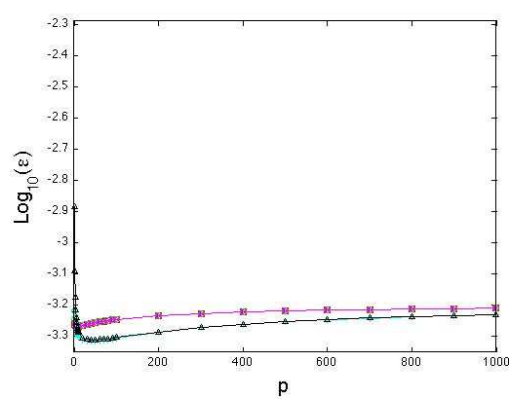

(c) $\Lambda_{L}(X)$ :p.const - $\xi(X)$ : p.linear

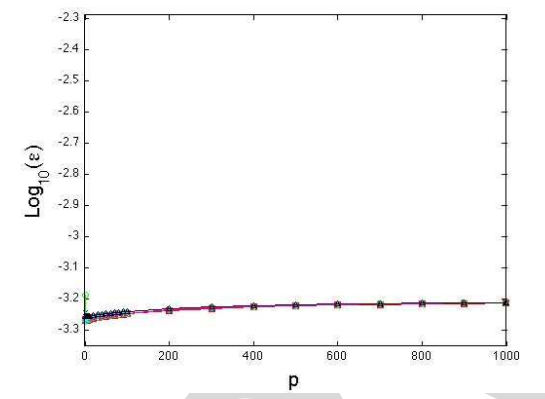

(b) $\Lambda_{L}(X):$ p.linear $-\xi(X)$ : p.cubic

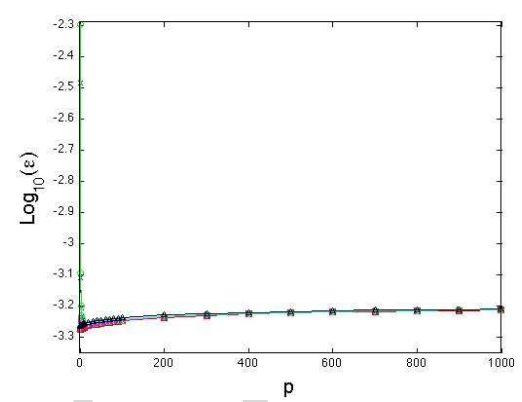

(d) $\Lambda_{L}(X)$ :p.const - $\xi(X)$ : p.cubic

Figure 13: Total error of the atomistic-to-continuum blended model as a function of the penalty parameter $p$, for different cases, for the constant-load case in a semi-log scale. The model choices and label interpretation are the same as in Fig. 5. As in Fig. 12, some curves overlap each other (see the caption of Fig. 12 for further details).
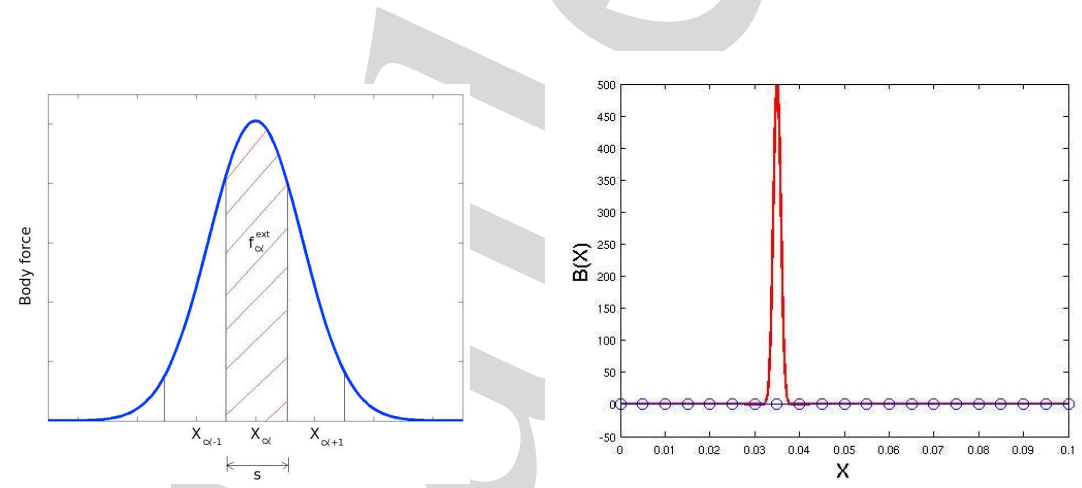

Figure 14: Left: relation between a continuous body force at the macroscale level and a microscopic atomistic external force; the atomistic force $f_{\alpha}^{\text {ext }}$, acting on an atom at $X_{\alpha}$, is taken as the integral of the body force over the Voronoi cell corresponding to the specific atom position, i.e., $\left[X_{\alpha}-s / 2, X_{\alpha}+s / 2\right]$, with $s$ the lattice spacing. Right: external singular force profile of a narrow Gaussian concentrated around a single atom. 
Table 2: Model parameters for the singular force simulations.

to the external force exerted on a particle at $X_{\alpha}$ is illustrated, and, on the right, the narrow general profile of a body force with a Gaussian form is shown, in the range $[0,0.1]$. Given a continuous force profile $\mathrm{B}(X)$, we determine the corresponding force $f_{\alpha}^{\text {ext }}$, acting on the particle located at $X_{\alpha}$, as the integral of the continuous body force over the Voronoi cell corresponding to $X_{\alpha}$, i.e., $\left[X_{\alpha}-s / 2, X_{\alpha}+s / 2\right]$, where $s$ denotes the lattice spacing; the expression for the force is as follows:

$$
f_{\alpha}^{\text {ext }}=\int_{X_{\alpha}-s / 2}^{X_{\alpha}+s / 2} \mathrm{~B}(X) d X \approx \sum_{k} \mathrm{~B}\left(X_{k}\right) w_{k}
$$

where the integral is approximated by some quadrature rule with appropriate accuracy.

To introduce a numerical example, we approximate a singular load by a very narrow Gaussian, having the form

$$
f(x)=\frac{1}{\sqrt{2 \pi} \sigma}\left(-\frac{(x-\mu)^{2}}{2 \sigma^{2}}\right),
$$

where $\mu$ determines the position of the center of the Gaussian and $\sigma$ its width. A quadrature rule is used to approximate the integrals of the external body force of the continuum contribution of the AtC blended model (cf. right-hand side of (2.11)) and the purecontinuum FE one, i.e.,

$$
\int_{X_{i}}^{X_{f}} \xi(X) \mathrm{B}(X) \omega_{j}^{h}(X) d X
$$

where $\xi(X) \equiv 1$ in the pure-continuum FE model. In order to avoid significant errors introduced in the conversion from the continuous force $\mathrm{B}(X)$ to its discrete version $f_{\alpha}^{\text {ext }}$, we use a high-order quadrature (64 points) to compute the atomistic force in (6.1). We would like to concentrate on the model performance around the center of the Gaussian, assumed to be in the atomistic domain outside the bridge region, i.e., $\Omega_{0}^{M} \backslash \Omega_{0}^{\text {bri }}$, and not on the model performance in the bridge region. Thus, taking into account the convergence studies in Section 5, we use appropriate AtC blended model choices that closely reproduce the pure-atomistic results; the choices are presented in Table 2, and a uniform LM grid is implemented. We apply a force concentrated around 1 particle as it is shown in Fig. 14 (right), where the blue circles represent the atomistic particles; the parameters of the Gaussian profile are $\sigma=0.0008$ and $\mu=0.035$. In Fig. 15, we compare the results, between the different models, for different choices for the order of the quadrature rule applied to the integrals containing the body force $\mathrm{B}(X)$, both in the pure-continuum $\mathrm{FE}$ and AtC blended ( $c f$. right-hand side of (2.11)) models; a comparison between the AtC 


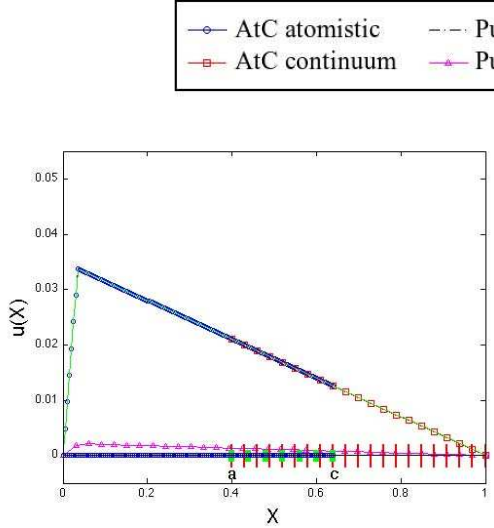

(a) Quadrature order $=5$

AtC error $=1.03 \mathrm{e}-15 ;$ FE error $=1.82 \mathrm{e}-2$

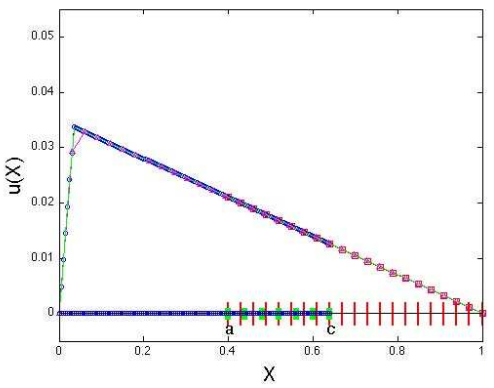

(c) Quadrature order $=32$ $\mathrm{AtC}$ error $=1.03 \mathrm{e}-15 ; \mathrm{FE}$ error $=4.18 \mathrm{e}-4$

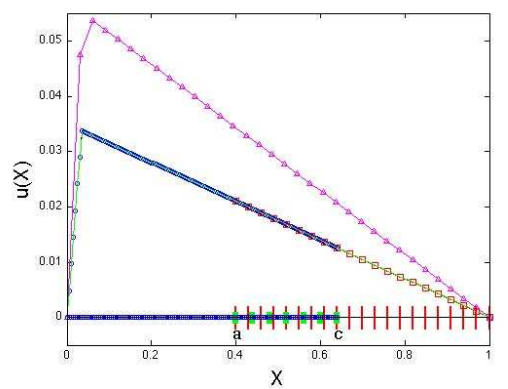

(b) Quadrature order $=10$

AtC error $=1.03 \mathrm{e}-15$; FE error $=1.22 \mathrm{e}-2$

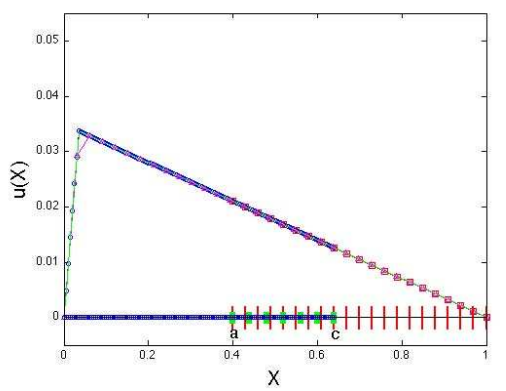

(d) Quadrature order $=64$; AtC error $=1.03 \mathrm{e}-15 ;$ FE error $=4.19 \mathrm{e}-4$

Figure 15: Comparison of the displacement profiles between the different models: atomistic-to-continuum (AtC) blended (with an atomistic solution in [0,0.64], and a continuum finite element (FE) approximation in $[0.4,1.0]$ ), pure-atomistic, pure-continuum FE, and the PDE $-K_{c} \frac{d^{2} u}{d X^{2}}=B(X)$, for different choices for the order of the quadrature rule applied to the integrals containing the body force $B(X)$ both in the pure-continuum $F E$ and AtC blended models. The AtC blended model and pure-continuum FE model errors are shown on each case. In addition to the displacements, the multiscale grid is shown in the plots, with the blue circles representing atoms, the thin red vertical bars FE nodes, and the thick green vertical bars Lagrange multiplier nodes.

blended model and pure-continuum FE model errors AtC blended model produces the same results as in the pure-atomistic model, and these results seem to reproduce the solution of the corresponding PDE; in contrast, the purecontinuum FE model does not reproduce the same results for a low quadrature order. The pure-continuum FE results become closer to the pure-atomistic results when we increase the order of the quadrature, although, even for a high-order quadrature, e.g., 64 points, the error is still large, i.e., $4.19 e-4$ (see Fig. 15(d)); the pure-continuum FE results

¥¥The error in the AtC blended model is computed using (3.1); the corresponding error in the purecontinuum FE model is computed, similarly, by the $L_{2}$-like norm for the difference between the pureatomistic model solution and the interpolation of the pure-continuum FE model solution. 


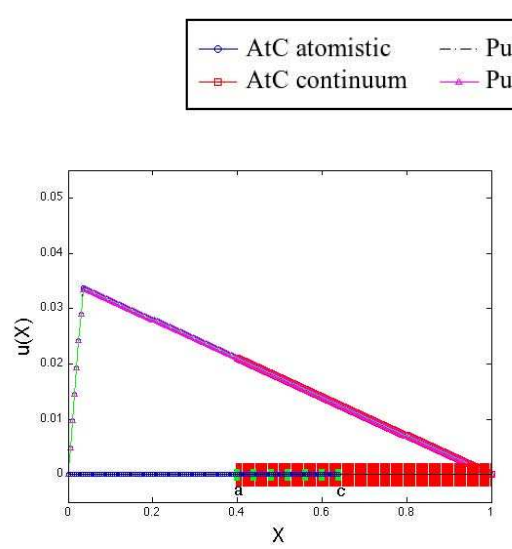

(a) Full resolution FE

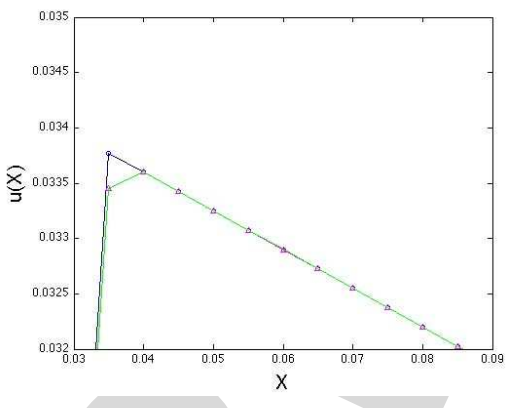

(b) Full resolution FE (zoom-in)

Figure 16: Comparison of the displacement profiles between different models (see caption of Fig. 15 for details), using a quadrature of 64 points (for the integrals containing the body force $B(X)$ both in the pure-continuum $\mathrm{FE}$ and AtC blended models) and an atomistic resolution for the FE simulation, i.e., $N_{u}=121$ (this corresponds to $201 \mathrm{FE}$ nodes in $[0,1]$ ). As in Fig. 15, the multiscale grid is shown in (a) in addition to the displacement profiles. The entire plot is shown in (a), whereas in (b) a zoom-in to the area of interest is presented.

error can be improved by increasing the number of FE nodes.

Using the same resolution for the FE grid as in the pure-atomistic model, i.e., $N_{u}=121$ (this corresponds to $201 \mathrm{FE}$ nodes in $[0,1]$ ), we get the results presented in Fig. 16. In (a), we see that all models seem to agree, although, when we zoom in (b), we see that the FE solution agrees with the PDE solution, but differs from the pure-atomistic and AtC blended model solutions (which agree each other). It seems that the quadrature used for the calculation of the atomistic force $f_{\alpha}^{\text {ext }}$ in (6.1), does not capture the exact profile of the continuum body force $\mathrm{B}(X)$; the error between the pure-continuum FE and the pureatomistic solutions is, in this case, $2.22 e-5$. If the interatomic spacing is such that we have 801 atoms in the entire domain, i.e., $[0,1]$, and still use the same resolution for the FE method as in the atomistic model (this resolution corresponds to a choice of $N_{d}=513$ and $N_{u}=481$ ), the error is reduced to $1.32 e-6$.

In general, we do not have the exact solution of the PDE, and the pure-atomistic solution is taken as the exact solution. Therefore, a more appropriate approach would be to start from a discrete force (i.e., the force profile in the atomistic region) and find a corresponding continuum expression; this approach is discussed in the next section.

\subsection{From a discrete to a continuous force}

In classical continuum mechanics, we refer to material objects as continuous, whereas this is considered an approximation, at the macroscopic level, of the underlying microscopic discrete view of matter. Under the accepted discrete approach, matter is described as composed of particles to which an external force is applied, and the continuum body 

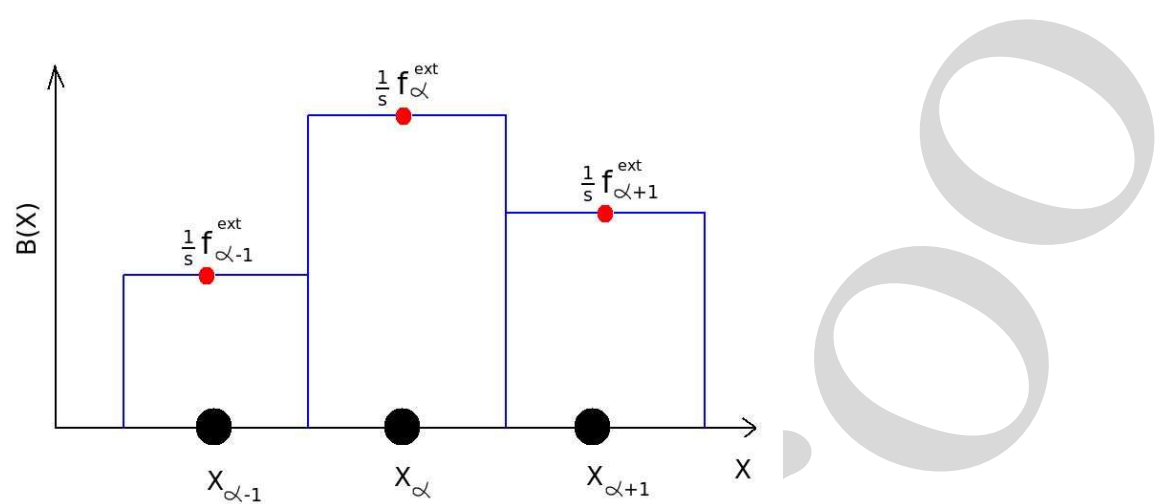

Figure 17: Piecewise constant continuous body force $\mathrm{B}(X)$ at the macroscale level, having values related to a given microscopic atomistic force; $f_{\alpha}^{\text {ext }}$ is the atomistic external force acting on a particle at $X_{\alpha} ; s$ is a uniform atomistic resolution, i.e., $s=X_{\alpha+1}-X_{\alpha}$.

force is an averaged force per unit volume; when the force is smooth at the microscopic level, then, the chosen averaging scale, at the macroscopic level, does not make too much difference, but when the force changes at the microscopic level, the averaging scale takes on an important role.

We are interested in applying a continuous model at a microscopic scale; thus, we need to know the correspondence between the atomistic force and the continuous body force applied on the system. We would like to discuss the case of a singular load (or more precisely, an external force applied to a few particles) and see how we can implement it on the continuous model. We approximate a singular load by a very narrow discrete Gaussian profile, assuming that the force acts only at the atomistic positions. In order to preserve the sharp force behavior, we want to compute the force average over as few atoms as possible. Therefore, we assume that the atom at $X_{\alpha}$ is a representative particle of the region $\left[X_{\alpha}-s / 2, X_{\alpha}+s / 2\right]$, with $s$ the lattice spacing, i.e., we divide our chain of atoms into Voronoi cells in the reference configuration, and assume each atom is the representative particle of its corresponding cell. We now calculate the body force acting in that cell as the force acting on the particle divided by the cell length, i.e., $\mathrm{B}(X)=f_{\alpha}^{\text {ext }} / \mathrm{s}$ for $X \in\left[X_{\alpha}-s / 2, X_{\alpha}+s / 2\right]$ The body force is then a piecewise constant function as it is shown in Fig. 17.

To obtain the smallest error, we use the atomistic resolution on the FE approximation, defining a FE node at each atomistic site; in this case, and assuming $\mathrm{B}(X)$ is piecewise constant, we can calculate the body force contribution to the FE equation as follows:

$$
\begin{aligned}
& \int_{X_{i}}^{X_{f}} \mathrm{~B}(X) \omega_{j}^{h}(X) d X=\int_{X_{\alpha-1}}^{X_{\alpha+1}} \mathrm{~B}(X) \omega_{\alpha}^{h}(X) d X \\
= & \frac{f_{\alpha-1}^{\text {ext }}}{s} \int_{X_{\alpha-1}}^{X_{\alpha}-s / 2} \omega_{\alpha}^{h}(X) d X+\frac{f_{\alpha}^{\text {ext }}}{s} \int_{X_{\alpha}-s / 2}^{X_{\alpha}+s / 2} \omega_{\alpha}^{h}(X) d X+\frac{f_{\alpha+1}^{\text {ext }}}{s} \int_{X_{\alpha}+s / 2}^{X_{\alpha+1}} \omega_{\alpha}^{h}(X) d X \\
= & \frac{1}{8}\left(f_{\alpha-1}^{\text {ext }}+6 f_{\alpha}^{\text {ext }}+f_{\alpha+1}^{\text {ext }}\right) \neq f_{\alpha}^{\text {ext }},
\end{aligned}
$$


assuming $j \equiv \alpha$ (i.e., the FE grid node $j$ is at the same position as the atom $\alpha$ ), and the FE and atomistic grids resolutions are identical, i.e., $h=s$. Therefore, we get a weighted average of $f_{\alpha-1}^{\text {ext }}, f_{\alpha}^{\text {ext }}$, and $f_{\alpha+1}^{\text {ext }}$. This treatment does not reproduce the pure-atomistic external force, i.e., $f_{\alpha}^{\text {ext }}$, and gives some smoothing of the force. In particular, if the force is a delta function or close to that, we get a smoothing of it that produces wrong results.

In Table 3, we compare the errors of the AtC blended and pure-continuum FE models with respect to the pure-atomistic case. The simulations use the parameters in Table 2, but implement a FE resolution identical to the atomistic one, i.e., $N_{u}=121$ (this corresponds to $201 \mathrm{FE}$ nodes in $[0,1]$ ), and a uniform LM grid. The external force is assumed to have a Gaussian profile with the parameters $\sigma=0.0008$ and $\mu=0.035$, and the atomistic force $f_{\alpha}^{\text {ext }}$ is calculated integrating the Gaussian profile, using a high-order quadrature (64 points), as in (6.1); then, the continuum body force is computed based on the values obtained for the atomistic force. In this case, i.e., piecewise constant $B(X)$, the AtC blended model gives better results than the pure-continuum FE model ( $c f$. left columns in Table 3); in other words, because of the averaging property of the pure-continuum model, the FE method cannot recover the atomistic behavior, even if the FE grid resolution is the same as the atomistic one.

Table 3: Comparison of the error for the atomistic-to-continuum ( $\mathrm{AtC}$ ) blended and the pure-continuum finite element (FE) methods for the different integration approximations for the body force.

\begin{tabular}{||c|c||c|c||}
\hline \multicolumn{2}{||c||}{ Piecewise constant $\mathrm{B}(X)$} & \multicolumn{2}{c||}{ Trapezoidal rule } \\
\hline AtC & Pure-continuum FE & AtC & Pure-continuum FE \\
\hline $2.14 \mathrm{e}-15$ & $4.40 \mathrm{e}-5$ & $2.14 \mathrm{e}-15$ & $1.35 \mathrm{e}-15$ \\
\hline
\end{tabular}

Taking into account that the force is applied only at the atomistic positions, we can instead approximate the integrals on the right-hand side of the continuum model using a trapezoidal rule over the atomistic sites, i.e.,

$$
\begin{aligned}
\int_{X_{i}}^{X_{f}} \mathrm{~B}(X) \omega_{j}^{h}(X) d X & =\int_{X_{i}}^{X_{f}} \mathrm{~B}(X) \omega_{\alpha}^{h}(X) d X \\
& \approx \sum_{\beta=1}^{N_{d}-1} \frac{s}{2}\left(\mathrm{~B}\left(X_{\beta}\right) \omega_{\alpha}^{h}\left(X_{\beta}\right)+\mathrm{B}\left(X_{\beta+1}\right) \omega_{\alpha}^{h}\left(X_{\beta+1}\right)\right) \\
& =\sum_{\beta=1}^{N_{d}-1} \frac{1}{2}\left(f_{\beta}^{\text {ext }} \omega_{\alpha}^{h}\left(X_{\beta}\right)+f_{\beta+1}^{\text {ext }} \omega_{\alpha}^{h}\left(X_{\beta+1}\right)\right)=\frac{f_{\alpha}^{\text {ext }}}{2}+\frac{f_{\alpha}^{\text {ext }}}{2}=f_{\alpha}^{\text {ext }},
\end{aligned}
$$

where we have used again a FE resolution equal to the atomistic one, reproducing the correct force expression. In Table 3, we compare the AtC blended and pure-continuum FE models errors with respect to the pure-atomistic case, with the FE resolution equal to the atomistic one. In this case, i.e., trapezoidal rule, the pure-continuum FE reproduces the atomistic external force (cf. right columns in Table 3).

A very important note is that although it seems that computing the right-hand side of the FE equations using the trapezoidal rule over the atomistic sites, on a grid with 
a resolution equal to the atomistic one, can reproduce the correct results, this implies a knowledge at each time of the singularity position. In other words, the FE method cannot assure that correct results would be obtained by just refining the grid to the atomistic resolution alone; only if the computation is done for the force calculated at the positions where the singularity occurs, then a correct result achievement is possible. Therefore, even if we refine the grid to the microscopic resolution, but the FE nodes do not coincide with the atomistic sites (or we miss the exact location of the singularity), then the FE model will not produce results as accurate as those of the AtC model.

\section{Multiple-neighbor interaction}

In this section, we deal with systems presenting multiple-neighbor atomistic interactions, whereas keeping the linearity of the interaction type, i.e., we still use the linear elasticity/linear spring model. As part of the generalization of the number of neighbor interactions, we introduce new implementation challenges; we have to determine how to modify the force constants to make the atomistic and continuum models consistent between each other; this will be presented in Section 7.1. In addition, multiple-neighbor atomistic interactions require a special boundary treatment because atoms located close to the boundary have to interact, in principle, with atoms beyond the boundary; in Section 7.2, we propose different approaches to deal with Dirichlet boundary conditions.

\subsection{Adapting the force constant}

The implementation of a multiple-neighbor interaction introduces modifications in the atomistic expressions. The atomistic force balance equations are, for $\alpha=1,2, \cdots, N_{d}$ :

$$
-\sum_{\substack{\beta=\alpha+N_{\text {neig }} \\ \beta \neq \alpha}}^{\alpha+N_{\text {neig }}} \frac{\widetilde{K}_{a}}{\left|X_{\alpha}-X_{\beta}\right|}\left(d_{\beta}-d_{\alpha}\right)=f_{\alpha}^{\text {ext }},
$$

with $N_{\text {neig }}$ the number of one-sided near neighbor atomistic interactions, and $\widetilde{K}_{a}$ a generalized atomistic force constant ${ }^{\dagger}$.

In the following, we present two different approaches (a uniform and a nonuniform force constant) for the relation between the generalized atomistic force constant $\widetilde{K}_{a}$ and the nearest-neighbor force constant $K_{a}$; this is in order to keep the consistency between the multiple-neighbor atomistic interaction and the linear elasticity continuum model

${ }^{\mp}$ For the AtC blended model implementation, we include the interatomic interaction blending function $\theta_{\alpha, \beta}$ on the left-hand side, and the energy atomistic blending function $\theta\left(X_{\alpha}\right)$ on the right-hand side. Furthermore, $N_{d}$ represents the number of atoms in the atomistic region of the AtC blended model, i.e., $\Omega_{0}^{M}$; for the pureatomistic model, this is replaced by the total number of atoms in the entire domain, i.e., $\Omega$. 
implemented in previous sections ${ }^{\ddagger}$.

Uniform force constant. We compute the factor $\widetilde{K}_{a}$ for a case of a general number of interactions. Using the Taylor expansion for a general function $f(X \pm n h)$, under the assumption that $f$ is smooth enough, one easily deduces

$$
f^{\prime \prime}(X)=\frac{2}{h N_{\text {neig }}\left(N_{\text {neig }}+1\right)} \sum_{n=1}^{N_{\text {neig }}} \frac{1}{n h}[(f(X-n h)-f(X))+(f(X+n h)-f(X))]+\mathcal{O}\left(h^{2}\right) .
$$

We would like to approximate consistently the equation

$$
-K_{c} u^{\prime \prime}(X)=\mathrm{B}(X) \rightarrow-K_{a} d^{\prime \prime}\left(X_{\alpha}\right)=\frac{f_{\alpha}^{\text {ext }}}{s} .
$$

Therefore, taking $X \equiv X_{\alpha}, f\left(X_{\alpha}\right) \equiv u\left(X_{\alpha}\right)=d_{\alpha}, K_{a}=K_{c}, \mathrm{~B}\left(X_{\alpha}\right) h=f_{\alpha}^{\text {ext }}$, and $h=s$ so that the corresponding equation is

$$
-\frac{2 K_{a}}{N_{\text {neig }}\left(N_{\text {neig }}+1\right)} \sum_{\substack{\beta=\alpha-N_{\text {neig }} \\ \beta \neq \alpha}}^{\alpha+N_{\text {neig }}} \frac{1}{\left|X_{\alpha}-X_{\beta}\right|}\left(d_{\beta}-d_{\alpha}\right)=\mathrm{B}\left(X_{\alpha}\right) h=f_{\alpha}^{\text {ext }},
$$

617 we conclude $(c f .(7.1))$ that $\widetilde{K}_{a}=\frac{2 K_{a}}{N_{\text {neig }}\left(N_{\text {neig }}+1\right)}$, the same constant for each pair interaction ${ }^{\S}$.

Nonuniform force constant. We now choose to include a different constant for each neighbor interaction so that we again use a Taylor expansion to deduce

$$
\sum_{n=1}^{N_{\text {neig }}}\left(K_{n} n h\right) f^{\prime \prime}(X)=\sum_{n=1}^{N_{\text {neig }}} \frac{K_{n}}{n h}[(f(X-n h)-f(X))+(f(X+n h)-f(X))]+\mathcal{O}\left(h^{3}\right) .
$$

Assuming $K_{n} \equiv \widetilde{K}_{a, n}, \sum_{n=1}^{N_{\text {neig }}} n \widetilde{K}_{a, n}=K_{a}$, and the same assumptions as in the uniform force constant derivation, we can write

$$
-\sum_{n=1}^{N_{\text {neig }}} \frac{\widetilde{K}_{a, n}}{n h}\left[\left(u\left(X_{\alpha}-n h\right)-u\left(X_{\alpha}\right)\right)+\left(u\left(X_{\alpha}+n h\right)-u\left(X_{\alpha}\right)\right)\right]=\mathrm{B}\left(X_{\alpha}\right) h=f_{\alpha}^{\text {ext }} ;
$$

$\mp$ ॠe look for an appropriate functional form for the atomistic force constant $\widetilde{K}_{a}$, in the case of a multipleneighbor atomistic interaction, in order to match the continuum and atomistic models implemented in the system. We assume the pure-atomistic model is the underlying "correct" solution of our system, although for practical purposes, we decided to use in the multiple-neighbor atomistic interaction case, the same continuum model for the continuum region used in the case of a nearest-neighbor atomistic interaction; thus, we found the relation between the assumed continuum model constant $K_{c}=K_{a}$ and the one corresponding to the underlying multiple-neighbor atomistic model $\widetilde{K}_{a}$.

$\S$ This relation appears in [21] in the context of upscaling a nonlocal linear springs molecular dynamics model to the nonlocal continuum model peridynamics. 
we can assume that the force constants corresponding to different neighbor interactions satisfy the relation ${ }^{\mathbb{I}}$

$$
n \widetilde{K}_{a, n}=\widetilde{K}_{a, 1}=2 \widetilde{K}_{a, 2}=3 \widetilde{K}_{a, 3}=\frac{K_{a}}{N_{\text {neig }}} \quad \text { or } \quad \widetilde{K}_{a, n}=\frac{K_{a}}{n N_{\text {neig }}},
$$

resulting in the model equation

$$
-\sum_{\substack{\beta=\alpha-N_{\text {neig }} \\ \beta \neq \alpha}}^{\alpha+N_{\text {neig }}} \frac{\widetilde{K}_{a,|\beta-\alpha|}}{\left|X_{\alpha}-X_{\beta}\right|}\left(d_{\beta}-d_{\alpha}\right)=f_{\alpha}^{\text {ext }} .
$$

Notice that the atomistic force constant $\widetilde{K}_{a}$ in (7.1), was replaced by $\widetilde{K}_{a,|\beta-\alpha|}$ to emphasize the explicit dependence of the force constant on the specific neighbor interaction (i.e., nearest-neighbor, second nearest-neighbor, etc.).

\subsection{Boundary treatments}

Different approaches have been proposed in the literature for the implementation of boundary conditions in atomistic systems; some approaches include periodic, constantstress, constant-displacement and free boundary conditions [1, 2, 19, 23-25]. Boundary conditions intend to reduce surface artifacts appearing in simulations; for example, periodic boundary conditions emulate an infinite system, i.e., we assume we simulate a small fraction of a larger system, and we expect to mimic a bulk phase.

In our case, we are interested in comparing the AtC blended model, not just to the pure-atomistic, but also to the pure-continuum model; in our pure-continuum model we implement Dirichlet boundary conditions, i.e., we assume a displacement for the first and last FE nodes; we would like to use a similar assumption for our pure-atomistic and AtC blended models, i.e., assume a fixed displacement for the first and last atoms, and for the first atom and last FE node respectively. Taking into account that for multipleneighbor interactions our nonlocal atomistic model requires an assumption regarding the displacements of all atoms within a layer of the boundary, we need to find a way to overcome the lack of information when we only have the information regarding the first and last atoms (or the first atom and last FE node in the AtC blended model). Consider Fig. 18, that illustrates the situation on the left boundary of the atomistic domain of the AtC blended system (this is the case in the multiscale approach, whereas in a pureatomistic model we have to deal with both boundaries). We see that, for example, in the case of a second-neighbor interaction, atom number " 2 " is supposed to interact with the "ghost atom" number " 0 ;" the "ghost atoms" are represented by magenta circles in the figure, i.e., atoms with a position $X_{\alpha}<X_{i}$; thus, we need to find a way to treat this problem.

II A similar relation between force constants in a mass-spring system appears in [1,2] for a particular case of a two-neighbor interaction system; the nearest-neighbor constitutive constant $K_{1}$ is chosen to have twice the value of the second nearest-neighbor one $K_{2}$, i.e., $K_{1}=2 K_{2}$. 


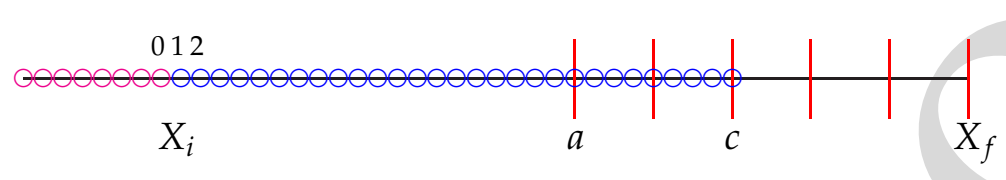

Figure 18: Atomistic-to-continuum coupling multiscale grid showing "ghost atoms". The domain of the system is $\left[X_{i}, X_{f}\right]$, and the bridge region is $[a, c]$. The red vertical bars in $\left[a, X_{f}\right]$ represent the finite element grid nodes. The blue circles in the region $\left[X_{i}, c\right]$ are the atoms in the atomistic region of our system. The magenta circles to the left of $X_{i}$ represent the "ghost atoms".

The general atomistic interaction expression is as follows:

$$
-\sum_{\substack{\beta=\alpha-N_{\text {neig }} \\ \beta \neq \alpha}}^{\alpha+N_{\text {neig }}} \frac{C(\alpha, \beta)}{\left|X_{\alpha}-X_{\beta}\right|}\left(d_{\beta}-d_{\alpha}\right)=f_{\alpha}^{\text {ext }}
$$

where $C(\alpha, \beta)$ represents the atomistic force constant, which depends on the model (see, e.g., below: truncation, asymmetry, adaptive, extended-BC, and ghost-atoms) and on the value of $\alpha$ and $\beta$. In the following, we introduce several approaches to deal with continuum Dirichlet-type boundary conditions, i.e., a displacement for the first and last particles of the system is assumed, in the presence of nonlocal atomistic interactions; in all cases we assume the atomistic model involves a one-sided $N_{\text {neig }}$ neighbor interactions, i.e., an atom inside the domain, and far from the boundaries, interacts with $N_{\text {neig }}$ neighbors on each side.

Truncation. We use an interaction with $N_{\text {neig }}$ neighbors on both sides (left and right), but avoid the interactions with atoms beyond the boundary, i.e., atoms with the index $\beta$ such that $\beta<1$ or $\beta>N_{d}$. Therefore,

$$
C(\alpha, \beta)=\left\{\begin{array}{cc}
0 & \beta<1 \text { or } \beta>N_{d} \\
\widetilde{K}_{a} & 1 \leq \beta \leq N_{d}
\end{array} \quad ; \quad \widetilde{K}_{a}=\frac{2 K_{a}}{N_{\text {neig }}\left(N_{\text {neig }}+1\right)} \quad \text { or } \quad \frac{K_{a}}{|\beta-\alpha| N_{\text {neig }}} .\right.
$$

Asymmetry. We allow for a different number of neighbor interactions to the left and right, depending on how close we are to the boundary. If we are far enough from both boundaries, we use an interaction with $N_{\text {neig }}$ neighbors on both sides; otherwise, we use an interaction with the number of neighbors available near the boundary, and with $N_{\text {neig }}$ neighbors towards the opposite side, changing the force constant accordingly. Thus, $C(\alpha, \beta)= \begin{cases}\widetilde{K}_{a}(\alpha-1) & \beta<\alpha \text { and }(\alpha-1)<N_{\text {neig }} \\ \widetilde{K}_{a}\left(N_{d}-\alpha\right) & \beta>\alpha \text { and }\left(N_{d}-\alpha\right)<N_{\text {neig }} ; \quad \widetilde{K}_{a}(N)=\frac{2 K_{a}}{N(N+1)} \quad \text { or } \frac{K_{a}}{|\beta-\alpha| N} . \\ \widetilde{K}_{a}\left(N_{\text {neig }}\right) & \text { otherwise }\end{cases}$

Adaptive. We use the same number of neighbor interactions to the left and right, using the minimum number of neighbors available between the left and right sides for the interaction. Therefore,

$$
C(\alpha, \beta)=\widetilde{K}_{a}(N) ; \quad \widetilde{K}_{a}(N)=\frac{2 K_{a}}{N(N+1)} \text { or } \frac{K_{a}}{|\beta-\alpha| N},
$$




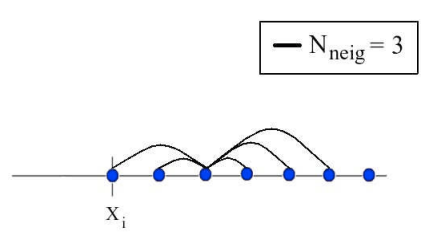

(a) Truncation

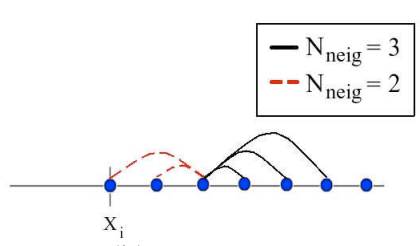

(b) Asymmetry

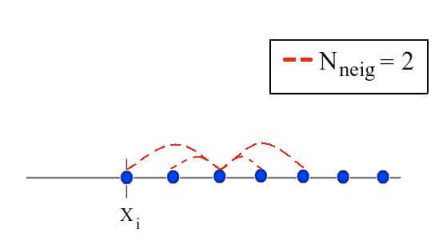

(c) Adaptive

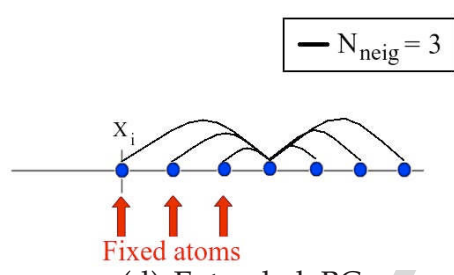

(d) Extended-BC

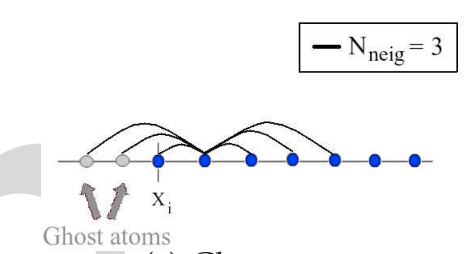

(e) Ghost-atoms

Figure 19: Comparison between the different boundary treatments. The pairwise interaction force constant $\widetilde{K}_{a}$ is computed for a specific choice of the number of one-sided atomistic neighbor interactions $N_{\text {neig. The red }}$ dashed lines represent interactions using a value of $N_{\text {neig }}=2$, whereas the black solid lines represent interactions using a value of $N_{\text {neig }}=3$.

with $N=\min \left(\alpha-1, N_{d}-\alpha, N_{\text {neig }}\right)$ the number of neighbor interactions.

Extended boundary conditions (extended-BC). We extend the boundary conditions inside the domain to the first $N_{\text {neig }}$ atoms closest to the boundary. Therefore, the interaction is implemented for atoms further than $N_{\text {neig }}$ atoms from the boundary. Thus,

$$
C(\alpha, \beta)=\left\{\begin{array}{ll}
\widetilde{K}_{a} & N_{\text {neig }}+1 \leq \alpha \leq N_{d}-N_{\text {neig }} \\
0 & \text { otherwise }
\end{array} ; \quad \widetilde{K}_{a}=\frac{2 K_{a}}{N_{\text {neig }}\left(N_{\text {neig }}+1\right)} \quad \text { or } \frac{K_{a}}{|\beta-\alpha| N_{\text {neig }}} .\right.
$$

Ghost-atoms. We use the general number of neighbor interactions $N_{\text {neig }}$ for every atom in the atomistic region. To solve the problem of the atoms near the boundaries, we add "ghost atoms" beyond the boundary and interact with them. The same boundary conditions are imposed on the "ghost atoms" as for the atoms on the boundaries. Therefore,

$$
C(\alpha, \beta)=\widetilde{K}_{a} ; \quad \widetilde{K}_{a}=\frac{2 K_{a}}{N_{\text {neig }}\left(N_{\text {neig }}+1\right)} \quad \text { or } \frac{K_{a}}{|\beta-\alpha| N_{\text {neig }}} .
$$

In Fig. 19, we present an illustration of the different boundary treatments for an atomistic interaction model with the choice of $N_{\text {neig }}=3$. The black solid lines represent interactions where the force constant is computed using the choice of $N_{\text {neig }}=3$, whereas the red dashed lines represent interactions where the force constant is computed using the choice of $N_{\text {neig }}=2$. The truncation method always uses the same force constant, but truncates the interactions beyond the boundary; the asymmetry method uses different constants for interactions to the left and to the right, depending on how close the particle 
is to the boundary; the adaptive method uses the same constant for interactions to the left and to the right, but the constant changes for different particles; the extended- $B C$ and ghost-atoms methods use the choice $N_{\text {neig }}=3$ for every pairwise interaction, but give a special treatment to certain particles or interactions in the system.

\subsubsection{Finite element interpolation for $\beta>N_{d}$}

In the AtC blended model, we need to deal with cases where a particle in the atomistic domain (including the bridge region) is supposed to interact with particles in the continuum domain beyond the bridge region; assume a particle $\alpha$ with a reference position $X_{\alpha} \in\left[X_{i}, c\right]$ is supposed to interact with a particle at the reference position $X_{\beta} \in\left(c, X_{f}\right]$, i.e., $\beta>N_{d}$, then, we replace the displacement $d_{\beta}$ in (7.1) with an interpolation of the FE displacements at $X_{\beta}$ (this is equivalent to the introduction of pad atoms [18,22] or ghost particles $[1,2]$ in $\Omega_{0}^{C} \backslash \Omega_{0}^{\text {bri }}$, with positions determined by the deformation of the continuum region where the atoms reside) as follows:

$$
\frac{\widetilde{K}_{a}}{\left|X_{\alpha}-X_{\beta}\right|}\left(d_{\beta}-d_{\alpha}\right)=\frac{\widetilde{K}_{a}}{\left|X_{\alpha}-X_{\beta}\right|}\left\{\left(\frac{X_{\beta}-X_{j}^{h}}{X_{j+1}^{h}-X_{j}^{h}}\right) u_{j+1}^{h}-\left(\frac{X_{\beta}-X_{j+1}^{h}}{X_{j+1}^{h}-X_{j}^{h}}\right) u_{j}^{h}\right\}-\frac{\widetilde{K}_{a}}{\left|X_{\alpha}-X_{\beta}\right|} d_{\alpha},
$$

where $X_{j+1}^{h}$ and $X_{j}^{h}$ are the positions of two FE nodes, such that $X_{\beta} \in\left[X_{j}^{h}, X_{j+1}^{h}\right]$.

\subsection{Results}

In this section, we present results for the various issues related to multiple-neighbor atomistic interactions.

Boundary treatment. We focus on the boundary treatment and see how the different approaches behave; for the simulations, the model choices are presented in Table 4. In Fig. 20, we present a comparison between the models for $N_{\text {neig }}=10$, in the case of the zero-load case (1st and 2nd columns) and the constant-load case (3rd and 4th columns); in each case we show the displacement profile (1st and 3rd columns) together with the strain profile (2nd and 4th columns); the computations were done using a uniform LM grid, and the uniform force constant choice (the results are qualitatively similar for the nonuniform force constant choice).

Table 4: Model parameters for the multiple-neighbor interactions simulations.

\begin{tabular}{||c|c|c|c|c|c|c|c|c|c|c|c|c||}
\hline$N_{d}$ & $N_{u}$ & $N_{\lambda}$ & $X_{i}$ & $a$ & $c$ & $X_{f}$ & $K_{c}$ & $K_{a}$ & $\xi(X)$ & $\Lambda_{L}(X)$ & Model & $\mathrm{p}$ \\
\hline 129 & 21 & 7 & 0.0 & 0.4 & 0.64 & 1.0 & 1.0 & 1.0 & cubic & linear & II & 1 \\
\hline
\end{tabular}

In order to understand the results from Fig. 20, we should take into account certain differences between the continuum (local) and the atomistic (nonlocal) models. Both the AtC blended model (on the right boundary) and the PDE (on both boundaries), treat the boundary following a local approximation, implementing the differential operator 


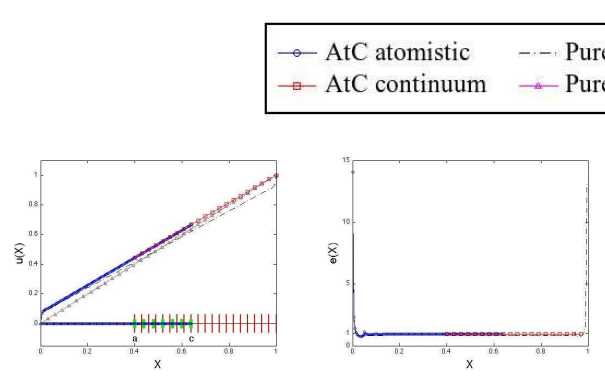

(a) Truncation (zero load)
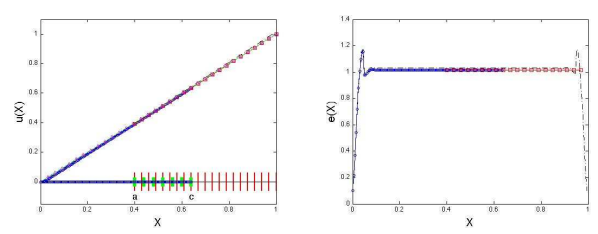

(c) Asymmetry (zero load)
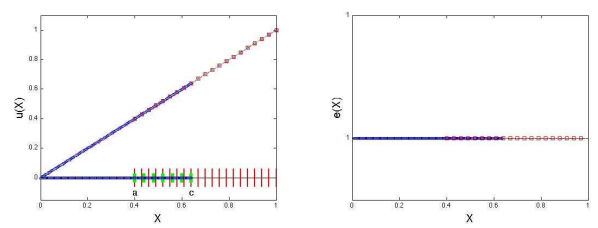

(e) Adaptive (zero load)
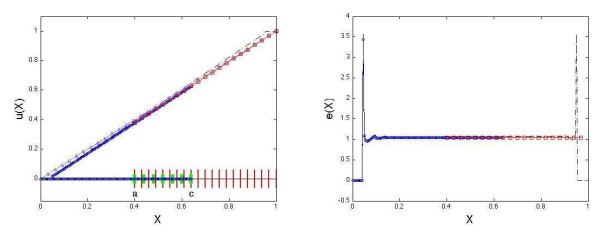

(g) Extended-BC (zero load)
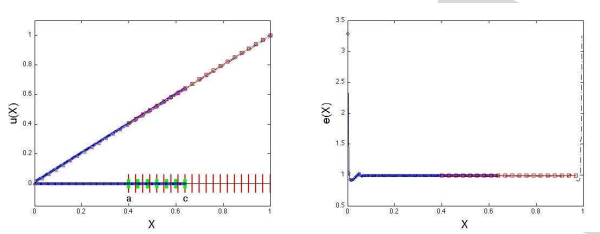

(i) Ghost-atoms (zero load)
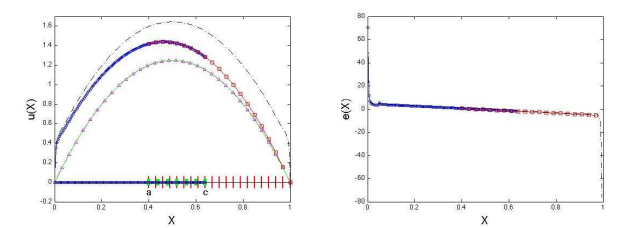

(b) Truncation (constant load)
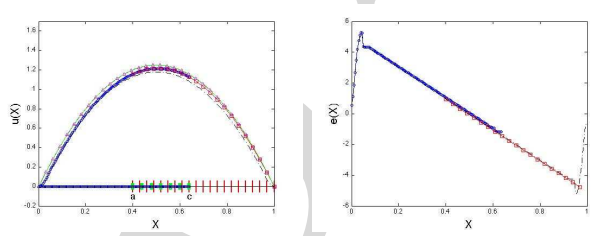

(d) Asymmetry (constant load)
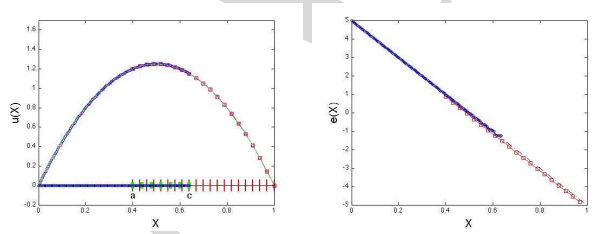

(f) Adaptive (constant load)
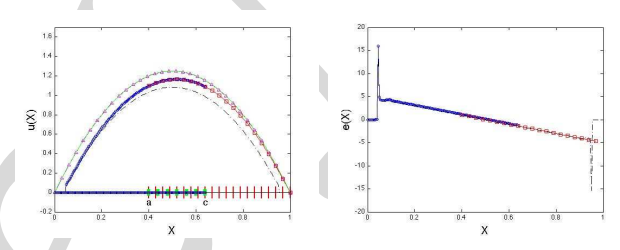

(h) Extended-BC (constant load)
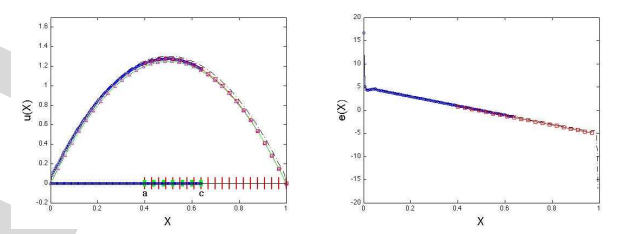

(j) Ghost-atoms (constant load)

Figure 20: Comparison of the displacements (1st and 3rd columns) and strains (2nd and 4th columns) for the case of the multiple-neighbor interaction, between the different boundary treatment methods: truncation, asymmetry, adaptive, extended-BC, and ghost-atoms. The simulations were run using the parameters presented in Table 4 with $N_{\text {neig }}=10$ and using a uniform choice for $\widetilde{K}_{a}$. Left columns (1st and 2 nd): zero-load case; right columns (3rd and 4th): constant-load case. For the displacement plots we compare the results between all the models: atomistic-to-continuum (AtC) blended (with an atomistic solution in $[0,0.64]$, and a continuum finite element (FE) approximation in $[0.4,1.0]$ ), pure-atomistic, pure-continuum FE and PDE $-K_{c} \frac{d^{2} u}{d X^{2}}=B(X)$, and in addition, we present the multiscale grid composed by atoms (blue circles), FE nodes (thin red vertical bars), and Lagrange multiplier grid nodes (thick green vertical bars). For the strain plots, we just compare the AtC blended and the pure-atomistic models. 
appearing in the PDE; the atomistic model (on both boundaries) and the AtC blended model (on the left boundary) treat the boundary following a nonlocal approximation. We have found particular expressions for the atomistic force constant in the nonlocal model, such that the behavior is consistent with the continuum model (see Section 7.1). We would expect to get similar profiles between atomistic and continuum expression, in the case both models treat the boundary in a consistent way.

The conclusions are as follows.

-It is expected that the AtC blended and the pure-atomistic models match on the left boundary, because they implement identical boundary treatments; similarly, the purecontinuum FE and the AtC blended models match on the right boundary.

- The adaptive method gives the closest profile to the pure-continuum FE solution (which reproduces the PDE solution); the reason for this is that it recovers a correct approximation for the second derivative for all the atoms. In contrast, the truncation and asymmetry methods do not provide a correct approximation to the differential operator in the continuum model, thus their solution deviates from the pure-continuum FE one; on the other hand, the asymmetry method gives a better approximation to the PDE than the truncation method, thus its solution is closer.

- It is interesting to note that the truncation and asymmetry approaches produce opposite curvatures on the boundaries, i.e., when the truncation approach gives a concave profile, the asymmetry gives a convex one, and vice versa.

- The ghost-atoms method reproduces pretty well the continuum results. Unfortunately, the lack of information regarding the correct boundary conditions for the "ghost atoms" introduces a deviation in the displacement profiles; introducing additional assumptions about their boundary conditions can improve the results.

- The extended- $B C$ method introduces the boundary conditions inside the domain, changing the profile accordingly.

Uniform vs. nonuniform $\widetilde{K}_{a}$. We now focus on the performance of the AtC coupling method in comparison to the pure-atomistic model, and compare between the uniform and nonuniform atomistic force constant choices. For that purpose, we want to get rid of the boundary effects and focus on the multiple-neighbor interactions inside the domain; we assume our system is far away from the boundaries, i.e., a very large system. To achieve that, we implement a variation of the ghost-atoms method, where we assume atoms close to the boundaries interact with "ghost atoms" beyond the boundaries, and impose the exact solution of the PDE $-K_{c} \frac{d^{2} u}{d X^{2}}=\mathrm{B}(X)$ to the "ghost atoms" displacements". In Fig. 21, we compare the error behavior of the AtC blended model in the case of multiple-neighbor atomistic interactions as a function of the number of one-sided atomistic neighbor interactions $N_{\text {neig, }}$, between the uniform and nonuniform choices for $\widetilde{K}_{a}$, for the zero-load (a) and constant-load (b) cases. The simulation parameters are presented in Table 4 . Both approaches for $\widetilde{K}_{a}$ reproduce the pure-atomistic solution in

"This "cheating" method was suggested to us by Michael L. Parks; the reason why it is called "cheating" is that, in general, the simulation results are not known a priori. 


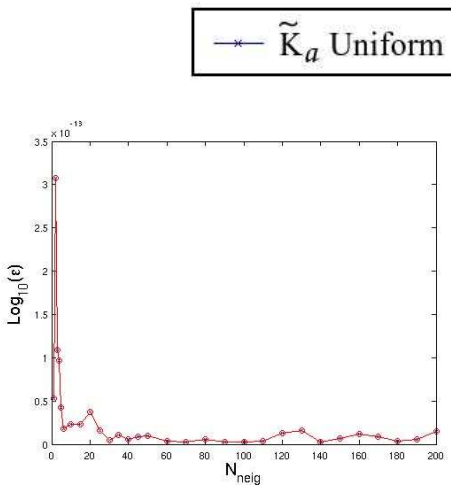

(a) Zero load

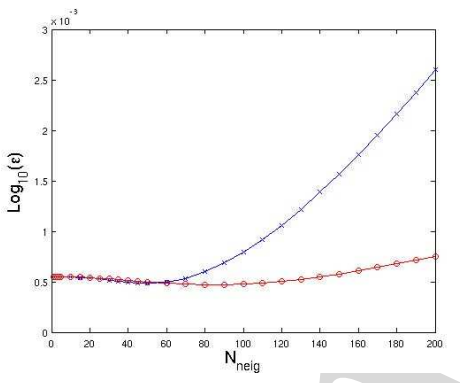

(b) Constant load

Figure 21: Comparison of the error behavior for different choices of number of one-sided atomistic neighbor

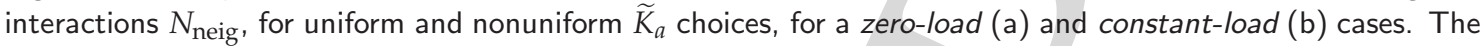
simulations were run using the parameters presented in Table 4.

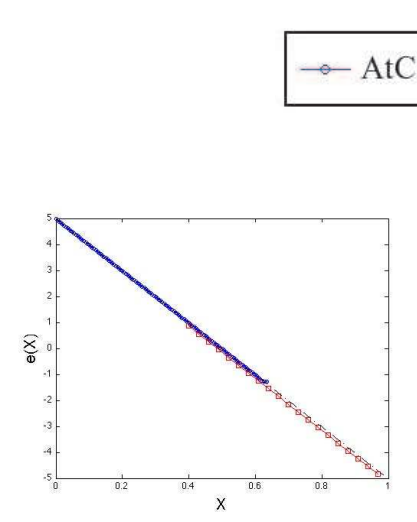

(a) $N_{\text {neig }}=1$

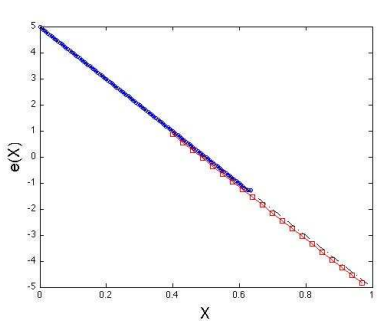

(d) $N_{\text {neig }}=1$

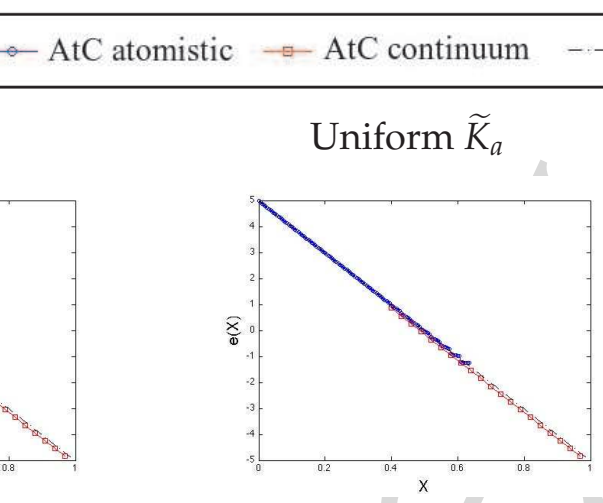

(b) $N_{\text {neig }}=20$

Nonuniform $\widetilde{K}_{a}$

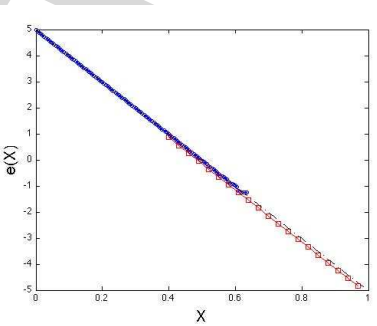

(e) $N_{\text {neig }}=20$

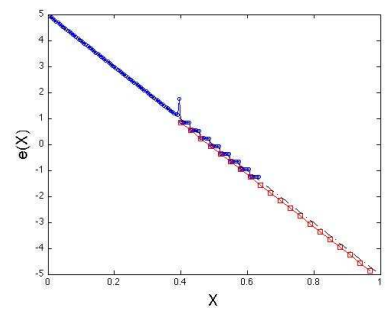

(c) $N_{\text {neig }}=200$

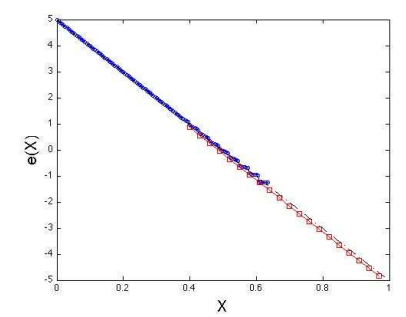

(f) $N_{\text {neig }}=200$

Figure 22: Comparison of the strain profiles between the atomistic-to-continuum ( $\mathrm{AtC}$ ) bended model (with an atomistic solution in $[0,0.64]$, and a continuum finite element approximation in $[0.4,1.0]$ ) and the pure-atomistic

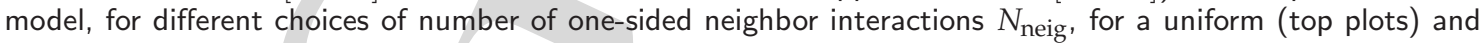
a nonuniform (bottom plots) $\widetilde{K}_{a}$ choice. The simulations used the parameters in Table 4 , for a constant-load case. 


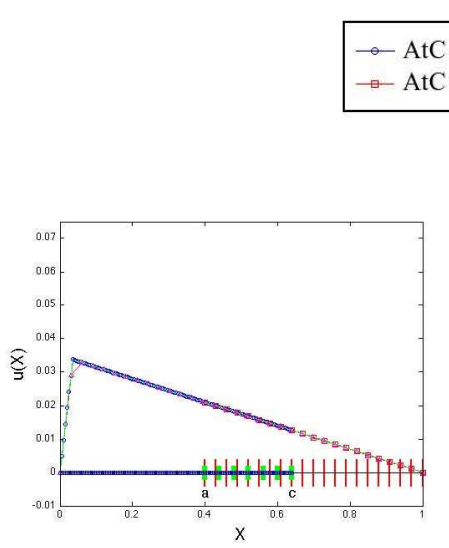

(a) $N_{\text {neig }}=1$

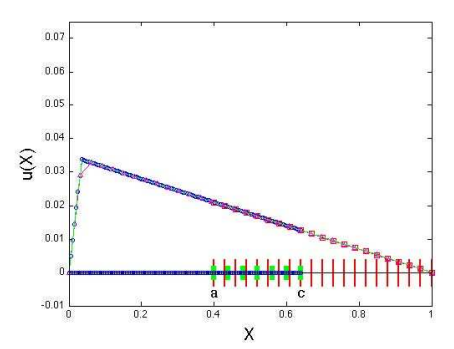

(d) $N_{\text {neig }}=1$

$\begin{array}{ll}\rightarrow \text { AtC atomistic } & \rightarrow-\text { Pure-atomistic } \\ \rightarrow \text { AtC continuum } & \rightarrow \text { Pure-continuum FE }\end{array}$

Uniform $\widetilde{K}_{a}$

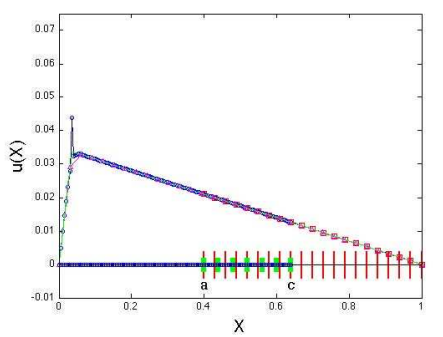

(b) $N_{\text {neig }}=5$

Nonuniform $\widetilde{K}_{a}$

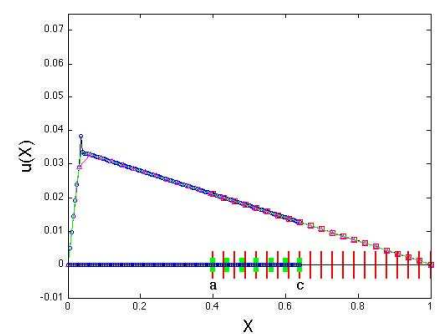

(e) $N_{\text {neig }}=5$

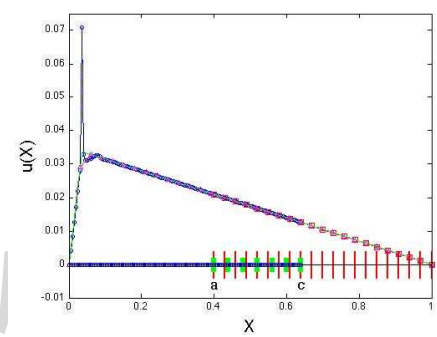

(c) $N_{\text {neig }}=10$

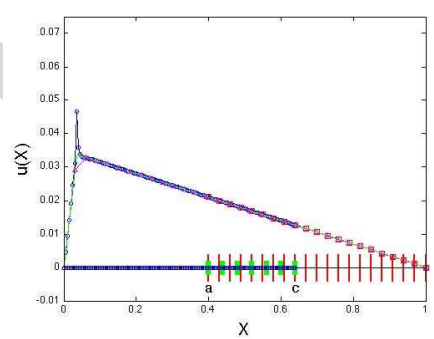

(f) $N_{\text {neig }}=10$

Figure 23: Comparison of the displacement profiles, for different choices of number of one-sided neighbor

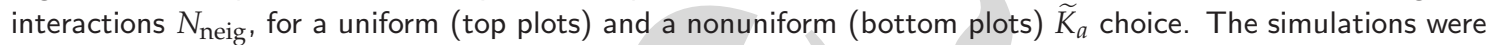
run using the parameters presented in Table 4. The plots compare the displacement profiles between different models and, in addition, present the multiscale grid (see Fig. 20 for further details regarding the models and the multiscale grid description).

the zero-load case (note the scale of the $y$-axis). In the constant-load case, the nonuniform $\widetilde{K}_{a}$ approach yields smaller errors for a large number of neighbor interactions.

In order to better understand the error behavior, we take a look at the strain profiles. In Fig. 22, we present a comparison between the uniform (top plots) and nonuniform (bottom plots) choices for $\widetilde{K}_{a}$, for the cases of $N_{\text {neig }}=1,20$, and 200, for the constant-load case, using the parameters in Table 4. The larger the number of neighbor interactions, the more step-wise the strain profile in the bridge region. On the other hand, this effect is stronger for the uniform choice for $\widetilde{K}_{a}$, giving a larger error; in addition, in that case, a spike develops at the left boundary of the bridge region.

Singular load case. We examine the effect of the multiple-neighbor interaction in the presence of a sharp external force. We use a Gaussian load with $\sigma=0.0008$ and $\mu=0.035$, presented in Fig. 14 (right plot). Fig. 23 presents a comparison between the uniform and 
nonuniform $\widetilde{K}_{a}$ choices, using different number of neighbor interactions; The simulations use the parameters presented in Table 4. We can see that the PDE solution** around the force location is different than the solution of the pure-atomistic and AtC blended models (which agree each other), for both choices of $\widetilde{K}_{a}$, for the multiple-neighbor case. In addition, the nonuniform $\widetilde{K}_{a}$ choice seems to smooth the effect appearing in the solution of the pure-atomistic and AtC blended models, in comparison to the uniform $\widetilde{K}_{a}$ choice.

\section{General conclusions}

We presented in this work a bridging technique application for coupling atomistic and continuum models. We explored a 1-D system and applied displacement constraints using an augmented Lagrange multiplier (LM) method. Several aspects of the model were analyzed using two different blending schemes (Model I and Model II).

As discussed in this paper, an implementation of different model component choices can improve the results of the atomistic-to-continuum (AtC) blended model, in relation to the pure-atomistic one. In particular, we studied the model dependence on the use of different choices for the energy blending functions (i.e., $\xi(X)$ for the continuum model and $\theta(X)=1-\xi(X)$ for the atomistic model): piecewise linear or piecewise cubic, the Lagrange multiplier (LM) basis functions $\Lambda_{L}(X)$ : piecewise linear or piecewise constant, the uniformity and resolution of the LM grid, the resolution of the finite element grid, the penalty parameter $p$, and the rule for the interatomic interaction blending function $\theta_{\alpha, \beta}$ : "average" or "midpoint".

Following the convergence studies of Section 5, we conclude as follows. Model II outperforms Model I, in most of the cases. For Model II, a uniform LM grid seems to be preferable, and, in addition, the piecewise cubic $\xi(X)$ choice appears as a good option in the case of small values of $p$, e.g., $p=1$; if, instead, we choose to use a piecewise linear $\xi(X)$ choice, a value for the penalty parameter of $p \approx 40$ appears to be optimal. For Model II, there seems to be little difference between the two choices for the LM basis functions $\Lambda_{L}(X)$. On the other hand, for Model I, a nonuniform LM grid with a piecewise linear LM basis functions $\Lambda_{L}(X)$ seems to be a good choice, in the case of small values of $p$, e.g., $p=1$. It is not completely clear, in Model I, which option for the energy blending function $\xi(X)$ is best; in the case of a piecewise linear $\xi(X)$ choice, there seems to exist an optimal value of $p \approx 10$ for the penalty parameter, and, in addition, the choice of the rule for the interatomic interaction blending function $\theta_{\alpha, \beta}$ does not affect the results; in the case of a piecewise cubic $\xi(X)$, the two options $\theta_{\alpha, \beta}$ yield similar results.

We also studied potential problems arising on the boundaries of the bridging region as well as a way to avoid them.

We gave some insight about the usefulness of using AtC coupling methods for simulations that try to capture singular phenomena. We showed a case of a singular load

\footnotetext{
** We assume $\mathrm{B}(X)=\frac{1}{\sqrt{2 \pi} \sigma} \exp \left(-\frac{(X-\mu)^{2}}{2 \sigma^{2}}\right)$, and solve analytically the PDE $-K_{c} \frac{d^{2} u}{d X^{2}}=\mathrm{B}(X)$; the exact solution
} of the PDE is used for comparison with the pure-atomistic, pure-continuum FE, and AtC blended models. 
modeled as a very narrow Gaussian, where the finite element method does not seem to be an appropriate numerical method for the solution; in particular, a knowledge of the singularity location at all times is required for a correct approximation of the continuum model. Moreover, we explained some difficulties arising in the description of the continuum concepts, such as the body force, when attempting to implement a continuum model at the atomistic scale.

Finally, we presented an approach to long-range interactions through a multipleneighbor atomistic force model. We have derived a functional form for the discrete model force constant that provides consistency between the atomistic and continuum models. Several approaches were proposed to treat Dirichlet-type boundary conditions, in the case of a multiple-neighbor interaction; some of the approaches implemented a variation of the specific functional form derived for the discrete model force constant. In addition, we showed some displacement deviations appearing in the multiple-neighbor interaction case in the presence of singular loads.

This work has focused in the study of the different aspects of the computational implementation of a one-dimensional AtC model. While a linear interaction model was considered here, the mathematical analysis of the various aspects and approaches introduced in this paper serve as a guidance for the implementation of more realistic systems.

\section{Acknowledgments}

This work was supported by the Department of Energy under grant number DE-FG0205ER25698. The authors thank Anter El-Azab for his valuable contributions in continuum mechanics concepts, Michael L. Parks for his comments regarding boundary treatments for atomistic models, John Burkardt for his support in the implementation of computational algorithms, and two anonymous referees for their helpful suggestions.

\section{References}

[1] S. Badia, P. Bochev, R. Lehoucq, M. Parks, J. Fish, M. Nuggehally, and M. Gunzburger, A force-based blending model for atomistic-to-continuum coupling; Inter. J. Multiscale Comp. Engrg. 5 (2007), 387-406.

[2] S. Badia, M. Parks, P. Bochev, M. Gunzburger, and R. Lehoucq, On atomistic-to-continuum coupling by blending; Multiscale Model. Simul. 7(1) (2008), 381-406.

[3] T. Belytschko and S. P. Xiao, Coupling methods for continuum model with molecular model; Intern. J. Multi. Comput. Engrg. 1(1) (2003), 115-126.

[4] J. Fish. M. A. Nuggehally, M. S. Shephard, and C. R. Picu, S. Badia, M. L. Parks, and M. Gunzburger, Concurrent AtC coupling based on a blend of the continuum stress and the atomistic force; Comput. Methods Appl. Mech. Engrg. 196 (2007), 4548-4560.

[5] H. Ben Dhia, Multiscale mechanical problems: the Arlequin method; C. R. Acad. Sci. Paris, Série IIlb, 326, (1998) 899-904. 
[6] H. Ben Dhia, Numerical modeling for multiscale problems: the Arlequin method, in: W. Wunderlich (Ed.), Proceedings of the First European Conference on Computational Mechanics, Muenchen, Germany, 1999.

[7] S. Prudhomme, H. Ben Dhia, P. T. Bauman, N. Elkhodja, and J. T. Oden, Computational analysis of modeling error for the coupling of particle and continuum models by the Arlequin method; Comput. Methods Appl. Mech. Engrg. 197 (2008) 3399-3409.

[8] W. E, B. Engquist, X. Li, W. Ren, and E. Vanden-Eijnden, Heterogeneous multiscale method: A review; Commun. Comput. Phys. 2(3) (2007), 367-450.

[9] R. E. Miller and E. B. Tadmor, A unified framework and performance benchmark of fourteen multiscale atomistic/continuum coupling methods; Modelling Simul. Mater. Sci. Eng. 17 (2009), 1-51

[10] J. Knap and M. Ortiz, An analysis of the quasicontinuum method; J. Mech. Phys. Solids 49 (2001), 1899-1923.

[11] J. Q. Broughton, F. F. Abraham, N. Bernstein, and E. Kaxiras, Concurrent coupling of length scales: Methodology and application; Phys. Rev. B 60(4) (1999), 2391-2403.

[12] F. F. Abraham, J. Q. Broughton, N. Bernstein, and E. Kaxiras, Spanning the length scales in dynamic simulation; Comput. Phys. 12(6) (1998), 538-546.

[13] E. B. Tadmor, M. Ortiz, and R. Phillips, Quasicontinuum analysis of defects in solids; Philos. Mag. A 73 (1996), 1529-1563.

[14] V. B. Shenoy, R. Miller, E. B. Tadmor, R. Phillips, and M. Ortiz, Quasicontinuum models of interfacial structure and deformation; Phys. Rev. Lett. 80(4) (1998), 742-745.

[15] S. T. O'Connell and P. A. Thompson, Molecular dynamics-continuum hybrid computations: A tool for studying complex fluid flows; Phys. Rev. E 52(6) (1995), R5792-R5795.

[16] E. G. Flekkøy, G. Wagner, and J. Feder, Hybrid model for combined particle and continuum dynamics; Europhys. Lett. 52(3) (2000), 271-276.

[17] X. B. Nie, S. Y. Chen, W. N. E, and M. O. Robbins, A continuum and molecular dynamics hybrid method for micro- and nano-fluid flow; J. Fluid Mech. 500 (2004), 55-64.

[18] W. A. Curtin and R. E. Miller, Atomistic/continuum coupling in computational materials science; Modelling Simul. Mater. Sci. Eng. 11 (2003), R33-R68

[19] W. K. Liu, E. G. Karpov, S. Zhang, and H. S. Park, An introduction to computational nanomechanics and materials; Comput. Methods Appl. Mech. Engrg. 193 (2004), 1529-1578.

[20] G. Lu and E. Kaxiras, Overview of Multiscale Simulations of Materials; Handbook of Theoretical and Computational Nanothechnology, Vol. X, pp. 1-33, edited by M. Rieth and W. Schommers (American Scientific Publishers, 2005).

[21] P. Seleson, M. L. Parks, M. Gunzburger, and R. B. Lehoucq, Peridynamics as an upscaling of molecular dynamics; Multiscale Model. Sim., accepted for publication, 2009.

[22] L. E. Shilkrot, R. E. Miller, and W. A. Curtin, Multiscale plasticity modeling: coupled atomistics and discrete dislocation mechanics; J. Mech. Phys. Solids 52 (2004), 755-787.

[23] D. C. Rapaport, The Art of Molecular Dynamics Simulation; Cambridge University Press 1995.

[24] V. V. Bulatov and W. Cai, Computer Simulations of Dislocations; Oxford University Press 2006.

[25] W. T. Ashurst and W. G. Hoover, Microscopic fracture studies in the two-dimensional triangular lattice; Phys. Rev. B 14(4) (1976), 1465-1473.

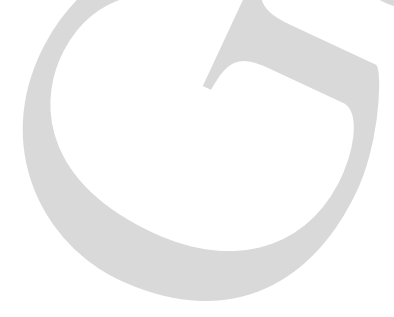

